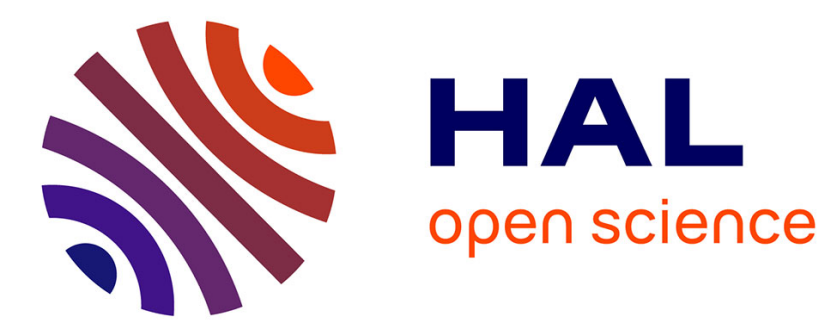

\title{
Discrete functional analysis tools for some evolution equations
}

Thierry Gallouët

\section{To cite this version:}

Thierry Gallouët. Discrete functional analysis tools for some evolution equations. Computational Methods in Applied Mathematics, 2018. hal-02078379

\section{HAL Id: hal-02078379 \\ https://hal.science/hal-02078379}

Submitted on 25 Mar 2019

HAL is a multi-disciplinary open access archive for the deposit and dissemination of scientific research documents, whether they are published or not. The documents may come from teaching and research institutions in France or abroad, or from public or private research centers.
L'archive ouverte pluridisciplinaire HAL, est destinée au dépôt et à la diffusion de documents scientifiques de niveau recherche, publiés ou non, émanant des établissements d'enseignement et de recherche français ou étrangers, des laboratoires publics ou privés. 


\title{
Thierry Gallouët \\ Discrete functional analysis tools for some evolution equations
}

\begin{abstract}
We present some discrete functional analysis tools for the proof of convergence of numerical schemes, mainly for equations including diffusion terms such as the Stefan problem or the Navier-Stokes equations in the incompressible and compressible cases. Some of the results covered here have been proved in previous works, coauthored with several coworkers.
\end{abstract}

Keywords: Discrete functional analysis, Stefan problem, Navier-Stokes equations

PACS: 65M08, 35Q30, 65M12

\section{Introduction}

The main purpose of this paper is to describe some tools, which have been recently developed, for the proof of convergence of numerical schemes for some evolution problems.

We have two main examples in mind.

The first example is the case of the Navier-Stokes equations, with the three classical particular cases, namely the incompressible case, the case of variable density and the compressible case. We briefly describe the most difficult case, which is the compressible case. The main unknowns are the density, $\rho$, the pressure, $p$, and the velocity, $\boldsymbol{u}$. They are function of the time $t$ which belongs to $[0, T]$ for a given $T>0$, and of the space variable $x$ which belongs to $\Omega$ where $\Omega$ is a given bounded open connected set of $\mathbb{R}^{d}, d=2$ or 3 . With a given source term, $f$, in the momentum equation, and a perfect gas equation of state (EOS for short), the equations read

$$
\begin{array}{r}
\partial_{t} \rho+\operatorname{div}(\rho \boldsymbol{u})=0, \\
\partial_{t}(\rho \boldsymbol{u})+\operatorname{div}(\rho \boldsymbol{u} \otimes \boldsymbol{u})-\Delta \boldsymbol{u}+\nabla p=f, \\
p=\rho^{\gamma} .
\end{array}
$$

The constant $\gamma$ is larger than 1 for $d=2$ and $3 / 2$ for $d=3$. To these equations we have to add some boundary condition, $\boldsymbol{u}=0$ for instance, and an initial condition on $\rho$ and $\boldsymbol{u}$ (or on $\rho \boldsymbol{u}$ ).

Thierry Gallouët, Université d'Aix-Marseille 
Considering the most interesting case $d=3$, with a convenient discretization of these equations, for instance with the so-called Marker-And-Cell scheme, MAC scheme for short, see [HW65] for the seminal paper, it is possible to obtain an $L^{\infty}(] 0, T\left[, L^{\gamma}(\Omega)\right)$-estimate on $\rho_{n}$ and an $L^{2}(] 0, T\left[, L^{6}(\Omega)^{3}\right)$-estimate on $\boldsymbol{u}_{n}$, where $\left(\rho_{n}, p_{n}, \boldsymbol{u}_{n}\right)_{n \in \mathbb{N}}$ is a sequence of approximate solutions (with a mesh size and a time step vanishing as $n \rightarrow+\infty)$. Then, up to a subsequence, it is possible to assume that, as $n \rightarrow+\infty, \rho_{n}$ weakly converges to some $\rho$ in $L^{2}(] 0, T\left[, L^{\gamma}(\Omega)\right)$ and $\boldsymbol{u}_{n}$ weakly converges to some $u$ in $L^{2}(] 0, T\left[, L^{6}(\Omega)^{3}\right)$. A first difficulty is that this weak convergence is not sufficient to prove the convergence of the product $\rho_{n} \boldsymbol{u}_{n}$ to $\rho \boldsymbol{u}$ (and of $\rho_{n} \boldsymbol{u}_{n} \otimes \boldsymbol{u}_{n}$ to $\rho \boldsymbol{u} \otimes \boldsymbol{u}$, note that, since $\gamma>3 / 2$, one has $\rho_{n} \boldsymbol{u}_{n} \otimes \boldsymbol{u}_{n}$ bounded in $L^{1}(] 0, T\left[, L^{r}(\Omega)^{3 \times 3}\right)$ for some $r>1$.). We tackle this difficulty in this paper (at least if $\gamma \geq 2$ for $\rho_{n} \boldsymbol{u}_{n}$ and $\gamma \geq 3$ for $\rho_{n} \boldsymbol{u}_{n} \otimes \boldsymbol{u}_{n}$ ). Another difficulty, not solved by the present paper, is to pass to the limit in the EOS (see, for instance, [GHLM17] for the stationary case).

The second example is the Stefan Problem. The set $\Omega$ is a given bounded open set of $\mathbb{R}^{d}, d \geq 1$. The function $\varphi$ is a Lipschitz continuous function from $\mathbb{R}$ to $\mathbb{R}$, nondecreasing and such that $\varphi^{\prime}=0$ on $] a, b[$, for some real numbers $a$, $b$ with $a<b$. We also assume that $\liminf _{|s| \rightarrow+\infty}|\varphi(s)| /|s|>0$. The unknowns are the functions $\rho$ and $u$, from $\Omega \times] 0, T[$ to $\mathbb{R}$, and the equation is

$$
\partial_{t} \rho-\Delta u=0, u=\varphi(\rho) .
$$

Here also, we have to add a boundary condition, $u=0$, and an initial condition on $\rho$.

Numerous discretizations of this problem, for instance with Finite Volumes Methods (see [EGH00]) or Gradient Discretization Methods (see [DEG $\left.{ }^{+} 17\right]$ ), lead to an $L^{2}(] 0, T\left[, L^{2}(\Omega)\right)$-estimate on a sequence of approximate solutions $\left(u_{n}, \rho_{n}\right)_{n \in \mathbb{N}}$. Then, one has, up to a subsequence, that $\rho_{n} \rightarrow \rho$ weakly in $L^{2}(] 0, T[$, $\left.L^{2}(\Omega)\right)$ and $u_{n} \rightarrow u$ weakly in $L^{2}(] 0, T\left[, L^{2}(\Omega)\right)$, as $n \rightarrow+\infty$.

With a mesh size and a time step vanishing as $n \rightarrow+\infty$, it is quite easy to prove that $\partial_{t} \rho-\Delta u=0$, in the distributional sense, but the difficulty is to prove that $u=\varphi(\rho)$. The main step for proving $u=\varphi(\rho)$ is to prove that $\lim _{n \rightarrow+\infty} \int_{0}^{T} \int_{\Omega} \rho_{n} u_{n} d x d t=\int_{0}^{T} \int_{\Omega} \rho u d x d t$. Then, by applying e.g. Minty's trick, one obtains the desired identity. This will be detailed in Sections 3.1.2 (continuous setting) and 3.2.2 (discrete setting).

The common feature of these two examples is that we have two sequences, namely $\left(\rho_{n}\right)_{n \in \mathbb{N}}$ and $\left(u_{n}\right)_{n \in \mathbb{N}}$, weakly converging in $L^{2}(] 0, T\left[, L^{q}(\Omega)\right)$ for a sequence and in $L^{2}(] 0, T\left[, L^{p}(\Omega)\right)$ for the other sequence, with $p, q>1,(1 / p)+$ $(1 / q)=1$. We denote by $\rho$ and $u$ the weak limits of these two sequences. Then, the objective is to present some convenient additional hypothesis in order to 
prove that, with $Q=\Omega \times] 0, T[$,

$$
\lim _{n \rightarrow+\infty} \int_{Q} \rho_{n}(y) u_{n}(y) d y=\int_{Q} \rho(y) u(y) d y,
$$

and to show that this additional hypothesis is satisfied in the case of the two examples described above.

For the sake of clarity, we will first consider the simple case corresponding essentially to the case of stationary equations (such as the stationary compressible Stokes equations). Then, we will consider the more difficult case of evolution equations, including the Stefan problem or the Navier-Stokes equations, where the time derivative plays a different role than the space derivatives.

As usual, the discrete analysis follows closely the continuous analysis and for this reason we begin by the tools in the continuous setting.

This paper uses ideas which were developed in some previous papers written in collaboration with several authors, among whom Jérôme Droniou, Robert Eymard, Raphaèle Herbin and Jean-Claude Latché. Part of the results given in this paper were presented in [Gal17].

\section{Stationary case}

\subsection{Continuous setting}

Let $\Omega$ be a bounded open set of $\mathbb{R}^{d}(d \geq 1), p, q>1,(1 / p)+(1 / q)=1$, and $\left(\rho_{n}\right)_{n \in \mathbb{N}}$ and $\left(u_{n}\right)_{n \in \mathbb{N}}$ be sequences such that $\rho_{n} \rightarrow \rho$ weakly in $L^{q}(\Omega), u_{n} \rightarrow u$ weakly in $L^{p}(\Omega)$ as $n \rightarrow+\infty$.

In general, one does not have, as $n \rightarrow+\infty$,

$$
\int_{\Omega} \rho_{n} u_{n} d x \rightarrow \int_{\Omega} \rho u d x .
$$

But, as it is well known, (5) is true if the sequence $\left(u_{n}\right)_{n \in \mathbb{N}}$ is bounded in $H_{0}^{1}(\Omega)$ and $p<2^{\star}$, with $2^{\star}=+\infty$, if $d=1$ or 2 , and $2^{\star}=2 d /(d-2)$ if $d \geq 3$ (note that $2^{\star}=6$ if $d=3$ ).

This can be proved in two slightly different ways.

The first way is to use the compactness of the sequence $\left(u_{n}\right)_{n \in \mathbb{N}}$ (note that, in this paper, "compactness" always means "strong compactness"). Indeed, since $H_{0}^{1}(\Omega)$ is compactly embedded in $L^{p}(\Omega)$, one has $u_{n} \rightarrow u$ in $L^{p}(\Omega)$ and then (5) holds. 
The second way is to use the compactness of the sequence $\left(\rho_{n}\right)_{n \in \mathbb{N}}$ in $H^{-1}(\Omega)$. Since $H_{0}^{1}(\Omega)$ is compactly embedded in $L^{p}(\Omega)$, by duality $L^{q}(\Omega)$ is compactly embedded in the dual space of $H_{0}^{1}(\Omega)$, namely $H^{-1}(\Omega)$. Here, one has identified, as usual, an element $f$ of $L^{q}(\Omega)$ with the element of $L^{p}(\Omega)^{\prime}$ defined by $g \mapsto \int_{\Omega} f g d x$. Then, one has

$$
\rho_{n} \rightarrow \rho \text { in } H^{-1}(\Omega) \text {, as } n \rightarrow+\infty \text {. }
$$

Since $\left(u_{n}\right)_{n \in \mathbb{N}}$ is bounded in $H_{0}^{1}(\Omega)$, one has also (without extraction of a subsequence)

$$
u_{n} \rightarrow u \text { weakly in } H_{0}^{1}(\Omega), \text { as } n \rightarrow+\infty .
$$

Using (6) and (7) we obtain

$$
\int_{\Omega} \rho_{n} u_{n} d x=\left\langle\rho_{n}, u_{n}\right\rangle_{H^{-1}, H_{0}^{1}} \rightarrow\langle\rho, u\rangle_{H^{-1}, H_{0}^{1}}=\int_{\Omega} \rho u d x .
$$

Remark 1. Another way to use the compactness of the sequence $\left(\rho_{n}\right)_{n \in \mathbb{N}}$ in $H^{-1}(\Omega)$ is to use, for all $n$, the solution of the Dirichlet problem with $\rho_{n}$ as datum, namely the function $w_{n}$ such that

$$
w_{n} \in H_{0}^{1}(\Omega), \int_{\Omega} \nabla w_{n} \cdot \nabla v d x=\int_{\Omega} \rho_{n} v d x \text { for all } v \in H_{0}^{1}(\Omega) .
$$

Using the weak convergence of $\rho_{n}$ in $L^{q}(\Omega)$, one has $w_{n} \rightarrow w$ in $H_{0}^{1}(\Omega)$ where $w$ is the solution of

$$
w \in H_{0}^{1}(\Omega), \int_{\Omega} \nabla w \cdot \nabla v d x=\int_{\Omega} \rho v d x \text { for all } v \in H_{0}^{1}(\Omega) .
$$

Indeed, it is quite easy to prove that $w_{n}$ weakly converges to $w$ in $H_{0}^{1}(\Omega)$. Then, taking $v=w_{n}$ in (9) and using the convergence of $\rho_{n}$ to $\rho$ in $H^{-1}(\Omega)$, one has

$$
\lim _{n \rightarrow+\infty} \int_{\Omega} \nabla w_{n} \cdot \nabla w_{n} d x=\lim _{n \rightarrow+\infty} \int_{\Omega} \rho_{n} w_{n} d x=\int_{\Omega} \rho w d x=\int_{\Omega} \nabla w \cdot \nabla w d x .
$$

This proves the convergence of the $H_{0}^{1}(\Omega)$-norm of $w_{n}$ to the $H_{0}^{1}(\Omega)$-norm of $w$. This gives

$$
\begin{aligned}
\lim _{n \rightarrow+\infty} \int_{\Omega}\left(\nabla w_{n}-\nabla w\right) \cdot\left(\nabla w_{n}-\nabla w\right) d x=\lim _{n \rightarrow+\infty} \int_{\Omega} \nabla w_{n} \cdot \nabla w_{n} d x \\
\quad-2 \lim _{n \rightarrow+\infty} \int_{\Omega} \nabla w_{n} \cdot \nabla w d x+\int_{\Omega} \nabla w \cdot \nabla w d x=0,
\end{aligned}
$$


which proves that $w_{n}$ converges to $w$ in $H_{0}^{1}(\Omega)$ (as $\left.n \rightarrow+\infty\right)$. In order to conclude, we now use (7) and obtain

$$
\int_{\Omega} \rho_{n} u_{n} d x d x=\int_{\Omega} \nabla w_{n} \cdot \nabla u_{n} \rightarrow \int_{\Omega} \nabla w \cdot \nabla u d x=\int_{\Omega} \rho u d x,
$$

since $\nabla w_{n}$ converges in $L^{2}(\Omega)^{d}$ to $\nabla w$ and $\nabla u_{n}$ weakly converges in $L^{2}(\Omega)^{d}$ to $\nabla u$.

\subsection{Discrete setting}

The set $\Omega$ is always a bounded open set of $\mathbb{R}^{d}(d \geq 1)$ but adapted to a space discretization. For all $n \in \mathbb{N}$, one has $\rho_{n} \in L_{n}$ and $u_{n} \in H_{n}$, where $L_{n}$ and $H_{n}$ are finite dimensional spaces included in $L^{\infty}(\Omega)$. Let $p, q>1$ with $(1 / p)+(1 / q)=1$. We assume that the sequence $\left(u_{n}\right)_{n \in \mathbb{N}}$ weakly converges to $u$ in $L^{p}(\Omega)$ and the sequence $\left(\rho_{n}\right)_{n \in \mathbb{N}}$ weakly converges to $\rho$ in $L^{q}(\Omega)$. We also assume, as in Section 2.1 , that $p<2^{\star}$ and we want (as in Section 2.1) a convenient additional condition giving (5).

In the case of conforming discretizations (such as Finite Element Methods), namely when $H_{n} \subset H_{0}^{1}(\Omega)$, an easy condition is, as in Section 2.1, to assume that the sequence $\left(u_{n}\right)_{n \in \mathbb{N}}$ is bounded in $H_{0}^{1}(\Omega)$. We are interested here by the more involved case when $H_{n} \not \subset H_{0}^{1}(\Omega)$ but $H_{n}$ is equipped with a norm, depending on $n$, "close" to the $H_{0}^{1}$-norm. This is the case for Finite Volume Methods or Gradient Discretization Methods, see, for instance, [EGH00], [EGH10] and $\left[\mathrm{DEG}^{+} 17\right]$ for elliptic and parabolic equations and see [HW65] for the seminal paper on the Marker-And-Cell scheme for the Navier-Stokes equations. As in Section 2.1, we will present two different methods, using compactness of the sequence $\left(u_{n}\right)_{n \in \mathbb{N}}$ or compactness of the sequence $\left(\rho_{n}\right)_{n \in \mathbb{N}}$.

\subsubsection{Compactness of the sequence $\left(u_{n}\right)_{n \in \mathbb{N}}$}

We first consider the case of classical Finite Volumes with admissible meshes, as in [EGH00, Definition 9.1], see Fig. 1. Roughly speaking for $d=2$ or 3 (the case $d=1$ is simpler), a classical Finite Volumes mesh consists in a family of disjoints "control volumes" which are open polygonal (if $d=2$ ) or polyhedral (if $d=3$ ) convex subsets of $\Omega$ whose closures cover $\Omega$. The interface between two control volumes is contained in an hyperplane of $\mathbb{R}^{d}$. For each control volume $K$, a point $x_{K}$ is given (it is not necessarily the center of gravity of $K$ ). Such a 
mesh is "admissible" if the segment joining the points $x_{K}$ and $x_{L}$ is orthogonal to the interface $\sigma$ between the control volumes $K$ and $L$ (see Fig. 1).

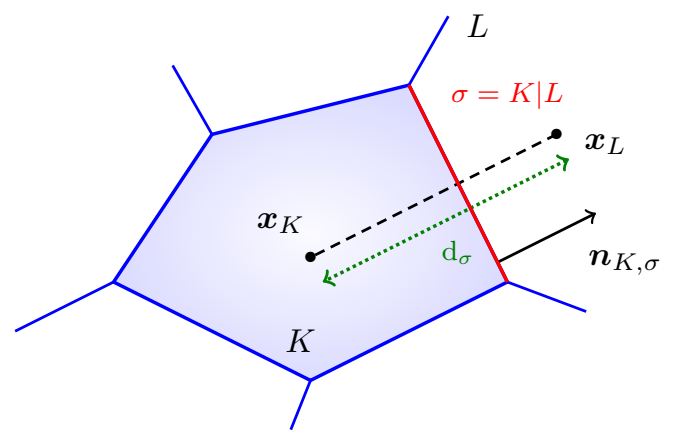

Fig. 1: Here is an example of admissible mesh in the sense of [EGH00]

In this case, the space $H_{n}$ is the space of functions which are constant on each control volume of the mesh $\mathcal{M}_{n}$. We denote by $h_{n}$ the maximum of the diameter of the control volumes of the mesh $\mathcal{M}_{n}$ and we assume that $\lim _{n \rightarrow+\infty} h_{n}=0$.

The space $H_{n}$ is equipped with a discrete norm which mimics the $H_{0}^{1}$-norm. We denote by $\mathcal{E}_{\text {int }}$ the interfaces which are in $\Omega$ and by $\mathcal{E}_{\text {ext }}$ the interfaces which are on the boundary of $\Omega$. If $\sigma$ is the interface between $K$ and $L$, we denote by $d_{\sigma}$ the distance between $x_{K}$ and $x_{L}$. If $\sigma$ is an interface of $K$ lying on the boundary of $\Omega, d_{\sigma}$ is the distance between $x_{K}$ and the boundary of $\Omega$. Finally, $m_{\sigma}$ denotes the $(d-1)$-Lebesgue measure of $\sigma$. With these notation, the norm on $H_{n}$, which mimics the $H_{0}^{1}$-norm, reads, if $u_{K}$ is the value of $u$ in the control volume $K$,

$$
\|u\|_{1,2, n}^{2}=\sum_{\sigma \in \mathcal{E}_{i n t}, \sigma=K \mid L} m_{\sigma} d_{\sigma}\left|\frac{u_{K}-u_{L}}{d_{\sigma}}\right|^{2}+\sum_{\sigma \in \mathcal{E}_{e x t}, \sigma \in \mathcal{E}_{K}} m_{\sigma} d_{\sigma}\left|\frac{u_{K}}{d_{\sigma}}\right|^{2} .
$$

We assume that the sequence $\left(\left\|\left(u_{n}\right)\right\|_{1,2, n}\right)_{n \in \mathbb{N}}$ is bounded. Then, it is proven in [EGH00, Theorem 9.3] that $u_{n} \rightarrow u$ in $L^{2}(\Omega)$. Now, we assume furthermore that $d_{K, \sigma} / d_{\sigma}$ is bounded from below by a positive number (independently of $K$ and $n$ ), where $d_{K, \sigma}$ is the distance between $x_{K}$ and $x_{\sigma}$, the point belonging to $\sigma$ and the segment joining $x_{K}$ and $x_{L}(\sigma=K \mid L)$. Then, it is also proven in [EGH00, Lemma 3.5] that the sequence $\left(u_{n}\right)_{n \in \mathbb{N}}$ is bounded in $L^{r}(\Omega)$ for any $r<+\infty$, if $d=1$ or 2 , and for $r=6$ if $d=3$. It is the so-called discrete Sobolev Embedding. Then $u_{n} \rightarrow u$ in $L^{p}(\Omega)$ since $p<2^{\star}$. Finally, since $\rho_{n} \rightarrow \rho$ weakly in $L^{q}(\Omega)$ (and $q=p /(p-1))$ we obtain (5) as desired. 
The fact that $u_{n} \rightarrow u$ in $L^{2}(\Omega)$ (and therefore in $L^{p}(\Omega)$ ) is a consequence of the Kolmogorov compactness theorem and of the following inequality, which is proven in [EGH00, Lemma 9.3] with some $C$ depending only on $\Omega$ and taking $u=0$ outside $\Omega$,

$$
\|u(\cdot+\eta)-u\|_{L^{2}\left(\mathbb{R}^{d}\right)} \leq C \sqrt{|\eta|}\|u\|_{1,2, n} \text { if } u \in H_{n} \text { and } \eta \in \mathbb{R}^{d} .
$$

In order to prove (11) the admissibility of the mesh, namely the orthogonality between the segment joining $x_{K}$ and $x_{L}$ and $K \mid L$ (the interface between $K$ and $L$ ), is used in the proof of [EGH00, Lemma 9.3]. Without this admissibility condition, we are not able to prove Inequality (11).

We consider now the case of non admissible meshes so that we do not assume the orthogonality condition between the segment joining the points $x_{K}$ and $x_{L}$ and the interface $\sigma$ between the control volumes $K$ and $L$ (see Fig. 1). But we assume (as before) that $d_{K, \sigma} / d_{\sigma}$ is bounded from below by a positive number (independently of $K$ and $n$ ). Then, we can also conclude by using an inequality on the translates on $u$ in the $L^{1}\left(\mathbb{R}^{d}\right)$-norm instead of the $L^{2}\left(\mathbb{R}^{d}\right)$-norm. This is done, for instance, in [EGH10]. Indeed, the $\|\cdot\|_{1,2, n}$-norm is the same as above (see [EGH10, norm defined by (43) or by (74) in Lemma 5.2]) and it is proven in [EGH10, Lemma 5.5] that

$$
\|u(\cdot+\eta)-u\|_{L^{1}\left(\mathbb{R}^{d}\right)} \leq|\eta| \sqrt{d}\|u\|_{1,2, n} \text { if } u \in H_{n} .
$$

Using again the Kolmogorov compactness theorem, we obtain the compactness of $\left(u_{n}\right)_{n \in \mathbb{N}}$ in $L^{1}(\Omega)$ and therefore $u_{n} \rightarrow u$ in $L^{1}(\Omega)$. But, the estimate on $\left\|u_{n}\right\|_{1,2, n}$ also gives an estimate on $u_{n}$ in $L^{r}(\Omega)$ for any $r<+\infty$, if $d=1$ or 2 , and for $r=6$ if $d=3$. This is again the discrete Sobolev embedding, given for instance in [EGH10, Lemma 5.3]. Using this estimate, together with the $L^{1}(\Omega)$ convergence, we can deduce the convergence of $u_{n}$ in $L^{p}(\Omega)$ ( since $p<2^{\star}$ ) and conclude as before that (5) holds.

\subsubsection{Compactness of the sequence $\left(\rho_{n}\right)_{n \in \mathbb{N}}$}

Although it is, of course, not necessary in this stationary case, we will try now to prove (5) by using compactness on $\rho_{n}$ instead of compactness of $u_{n}$. The interest of this second method will appear in the evolution case where the time variable plays a different role than that of the space variables. However, we will consider here only the case $p=q=2$, the general case $\left(p<2^{\star}, q=p /(p-1)\right)$ needs more work).

The main difficulty is that the bound on $u_{n}$ is on a norm which depends on $n$ (even if this norm is "close" to the $H_{0}^{1}$-norm). The trick we propose here 
(presented in [Gal17]) is to use the Sobolev space $H^{s}\left(\mathbb{R}^{d}\right)$ with some $\left.s \in\right] 0,1[$. We first recall the definition of the space $H^{s}\left(\mathbb{R}^{d}\right)$.

Definition 1. For $s \geq 0, H^{s}\left(\mathbb{R}^{d}\right)=\left\{u \in L^{2}\left(\mathbb{R}^{d}\right)\right.$ such that $\left.\left(1+|\cdot|^{2}\right)^{\frac{s}{2}} \hat{u} \in L^{2}\left(\mathbb{R}^{d}\right)\right\}$, where $\hat{u}$ is the Fourier transform of $u$. The norm for the space $H^{s}\left(\mathbb{R}^{d}\right)$ is given by

$$
\|u\|_{s}=\left\|\left(1+|\cdot|^{2}\right)^{\frac{s}{2}} \hat{u}\right\|_{L^{2}\left(\mathbb{R}^{d}\right)} .
$$

With this norm, $H^{s}\left(\mathbb{R}^{d}\right)$ is a Hilbert space.

Then, we set, for $s>0$,

$$
H^{s}=\left\{u \in H^{s}\left(\mathbb{R}^{d}\right), u=0 \text { a.e. on } \mathbb{R}^{d} \backslash \Omega\right\},
$$

equipped with the $H^{s}\left(\mathbb{R}^{d}\right)$-norm. The space $H^{s}$ is a Hilbert space as a closed subspace of the Hilbert space $H^{s}\left(\mathbb{R}^{d}\right)$.

We begin with the case of admissible meshes. Using (11), it is possible to prove that the sequence $\left(u_{n}\right)_{n \in \mathbb{N}}$ is bounded in $H^{s}$ for $0<s<\frac{1}{2}$. We give the proof of this result in Lemma 4 . Then, since $H^{s}$ is a Hilbert space (with its natural norm), we have $u_{n} \rightarrow u$ weakly in $H^{s}$. But, since $s>0$, we also have compactness of $H^{s}$ in $L^{2}(\Omega)$ since the $H^{s}$-norm of $u$ allows a control on the translates of $u$, see Lemma 5 . Then, by duality, identifying the space $L^{2}(\Omega)$ with its dual space, one has compactness of $L^{2}(\Omega)$ in $\left(H^{s}\right)^{\prime}$. This gives $\rho_{n} \rightarrow \rho$ in $\left(H^{s}\right)^{\prime}$ and we can conclude as in the continuous case, but with $H^{s}$ instead of $H_{0}^{1}$ :

$$
\int_{\Omega} \rho_{n} u_{n} d x=\left\langle\rho_{n}, u_{n}\right\rangle_{\left(H^{s}\right)^{\prime}, H^{s}} \rightarrow\langle\rho, u\rangle_{\left(H^{s}\right)^{\prime}, H^{s}}=\int_{\Omega} \rho u d x .
$$

In the case of non admissible meshes, we can also conclude but we need a little more work. We have to work with (12) instead of (11). We recall that, for $u \in H_{n}$, we also have an $L^{r}\left(\mathbb{R}^{d}\right)$-estimate (in term of $\|u\|_{1,2, n}$ ) on $u$ for any $r<+\infty$, if $d=1$ or 2 , and $r=6$ if $d=3$. Then, taking $r>2$, we use the inequality, for all $a>0$ and $\varepsilon>0$,

$$
a^{2} \leq \varepsilon a^{r}+\varepsilon^{\frac{-1}{r-2}} a .
$$

(Actually, this inequality follows from the fact that $a^{2}>\varepsilon a^{r}$ implies $a^{r-2}<\varepsilon^{-1}$ which implies $a<\varepsilon^{\frac{-1}{r-2}}$. Therefore, for all $\varepsilon>0$ and all $a>0$, one has $a^{2} \leq \max \left\{\varepsilon a^{r}, \varepsilon^{\frac{-1}{r-2}} a\right\}$.) Taking $\eta \in \mathbb{R}^{d}, a=|u(x+\eta)-u(x)|$ and integrating on $\mathbb{R}^{d}$ (recall that all functions are taken equal to 0 outside $\Omega$ ), we obtain, for $u \in H_{n}$,

$$
\|u(\cdot+\eta)-u\|_{L^{2}\left(\mathbb{R}^{d}\right)}^{2} \leq \varepsilon\|u(\cdot+\eta)-u\|_{L^{r}\left(\mathbb{R}^{d}\right)}^{r}+\varepsilon^{\frac{-1}{r-2}}\|u(\cdot+\eta)-u\|_{L^{1}\left(\mathbb{R}^{d}\right)} .
$$


It remains to choose $\varepsilon=|\eta|^{\frac{r-2}{r-1}}$. The $L^{r}\left(\mathbb{R}^{d}\right)$-estimate on $u$ (in terms of $\|u\|_{1,2, n}$ ) and (12) give the existence of $C$ depending only on $\Omega, r$ and the regularity of the mesh (to be more precise, we also use an homogeneity argument) such that

$$
\|u(\cdot+\eta)-u\|_{L^{2}\left(\mathbb{R}^{d}\right)} \leq C|\eta|^{\frac{r-2}{2(r-1)}}\|u\|_{1,2, n} \text { if } u \in H_{n} \text { and } \eta \in \mathbb{R}^{d} .
$$

In the case $d=3$, one takes $r=6$ and one has $\frac{r-2}{2(r-1)}=\frac{2}{5}$. It is now possible to conclude as in the case of admissible meshes. Using (15), the sequence $\left(u_{n}\right)_{n \in \mathbb{N}}$ is bounded in $H^{s}$ for $0<s<\frac{r-2}{2(r-1)}$ (see Lemma 4). Then, one has $u_{n} \rightarrow u$ weakly in $H^{s}$. Here also, since $s>0$, we have compactness of $H^{s}$ in $L^{2}(\Omega)$ (see Lemma 5$)$ and, by duality and identifying the space $L^{2}(\Omega)$ with its dual space, compactness of $L^{2}(\Omega)$ in $\left(H^{s}\right)^{\prime}$. This gives $\rho_{n} \rightarrow \rho$ in $\left(H^{s}\right)^{\prime}$ and we conclude with (14).

Remark 2. To conclude this section, we can also remark that it is possible, similarly to the continuous case, to use the compactness of $\left(\rho_{n}\right)_{n \in \mathbb{N}}$ under the form $\nabla_{n} w_{n} \rightarrow \nabla w$ in $L^{2}(\Omega)^{d}$ (and $\nabla_{n} u_{n} \rightarrow \nabla u$ weakly in $L^{2}(\Omega)^{d}$ ) where $\nabla_{n}$ is a discretization of $\nabla, w_{n}$ is the solution of a discrete equivalent of (9) and $w$ is the solution of (10). This method is used, for instance, in $\left[\mathrm{DEG}^{+} 17\right]$.

\section{Evolution case}

\subsection{Continuous setting}

Let $\Omega$ be a bounded open set of $\mathbb{R}^{d}(d \geq 1), T>0$ and $\left(\rho_{n}\right)_{n \in \mathbb{N}}$ and $\left(u_{n}\right)_{n \in \mathbb{N}}$ be sequences such that $\rho_{n} \rightarrow \rho$ weakly in $L^{2}(] 0, T\left[, L^{q}(\Omega)\right), u_{n} \rightarrow u$ weakly in $L^{2}(] 0, T\left[, L^{p}(\Omega)\right)$, with $1<p<2^{\star}$ and $q=p /(p-1)$ (recall that $2^{\star}=+\infty$, if $d=1$ or 2 , and $2^{\star}=2 d /(d-2)$ if $\left.d \geq 3\right)$. As in Section 2.1, in general one does not have

$$
\int_{0}^{T} \int_{\Omega} \rho_{n} u_{n} d x d t \rightarrow \int_{0}^{T} \int_{\Omega} \rho u d x d t, \text { as } n \rightarrow+\infty,
$$

even if $\left(u_{n}\right)_{n \in \mathbb{N}}$ is bounded in $L^{2}(] 0, T\left[, H_{0}^{1}(\Omega)\right)$ because there is no compactness of $L^{2}(] 0, T\left[, H_{0}^{1}(\Omega)\right)$ in $L^{2}(] 0, T\left[, L^{2}(\Omega)\right)\left(\right.$ or $L^{2}(] 0, T\left[, L^{2}(\Omega)\right)$ in $\left.L^{2}(] 0, T\left[, H^{-1}(\Omega)\right)\right)$. (We take here $p=q=2$ for simplicity.) Of course, (16) holds if $\left(u_{n}\right)_{n \in \mathbb{N}}$ is bounded in $H^{1}(] 0, T\left[, H_{0}^{1}(\Omega)\right)$ since there is compactness of $H^{1}(] 0, T\left[, H_{0}^{1}(\Omega)\right)$ in $L^{2}(] 0, T\left[, L^{2}(\Omega)\right)$. Similarly, (16) holds if $\left(\rho_{n}\right)_{n \in \mathbb{N}}$ is bounded in $H^{1}(] 0, T$, $L^{2}(\Omega)$ ) (and $\left(u_{n}\right)_{n \in \mathbb{N}}$ bounded in $L^{2}(] 0, T\left[, H_{0}^{1}(\Omega)\right)$ ) since there is compactness of $H^{1}(] 0, T\left[, L^{2}(\Omega)\right)$ in $L^{2}(] 0, T\left[, H^{-1}(\Omega)\right)$. (As usual, $L^{2}(\Omega)$ is identified to 
its dual space.) But such hypotheses are quite strong and the objective is to obtain (16) using weaker hypotheses on $\left(\partial_{t} u_{n}\right)_{n \in \mathbb{N}}$ or $\left(\partial_{t} \rho_{n}\right)_{n \in \mathbb{N}}$. We consider, for instance, the two examples given in Section 1.

\subsubsection{Compressible Navier-Stokes Equations}

Let $T>0, \Omega$ be a bounded open connected set of $\mathbb{R}^{d}, d=2$ or 3 and $\gamma \in \mathbb{R}$ such that $\gamma>1$ if $d=2, \gamma>3 / 2$ if $d=3$. For $n \in \mathbb{N}$, let $\left(\rho_{n}, p_{n}, \boldsymbol{u}_{n}\right)$ be a (weak) solution of (1)-(3) with $f_{n}$ as datum instead of $f$ and an homogeneous Dirichlet boundary condition on $\boldsymbol{u}_{n}$. We assume that $f_{n} \rightarrow f$ in $L^{2}(] 0, T\left[, L^{2}(\Omega)\right)$. Under a convenient hypothesis on the initial condition (on density and velocity), it is possible to obtain an $L^{\infty}(] 0, T\left[, L^{\gamma}(\Omega)\right)$-estimate on $\rho_{n}$ and an $L^{2}(] 0, T\left[, H_{0}^{1}(\Omega)^{d}\right)$ estimate on $\boldsymbol{u}_{n}$. Then, up to subsequences, it is possible to assume, as $n \rightarrow+\infty$, that $\rho_{n}$ weakly converges to some $\rho$ in $L^{2}(] 0, T\left[, L^{\gamma}(\Omega)\right)$ and $\boldsymbol{u}_{n}$ weakly converges to some $\boldsymbol{u}$ in $L^{2}(] 0, T\left[, H_{0}^{1}(\Omega)^{d}\right)$.

In order to pass to the limit on the equation $\partial_{t} \rho_{n}+\operatorname{div}\left(\rho_{n} \boldsymbol{u}_{n}\right)=0$ for proving that $\partial_{t} \rho+\operatorname{div}(\rho \boldsymbol{u})=0$ (in the distributional sense), the main difficulty is to prove that, for any $\varphi \in C_{c}^{\infty}\left(\mathbb{R}^{d} \times\right] 0, T[)$,

$$
\lim _{n \rightarrow+\infty} \int_{0}^{T} \int_{\Omega} \rho_{n} \boldsymbol{u}_{n} \cdot \nabla \varphi d x d t=\int_{0}^{T} \int_{\Omega} \rho \boldsymbol{u} \cdot \nabla \varphi d x d t .
$$

This is not easy since we only have weak convergence of $\rho_{n}$ and $\boldsymbol{u}_{n}$ in Lebesgue spaces.

Let $u_{n}$ be a component of the vector valued function $\boldsymbol{u}_{n}$ and consider that $d=3$ (the case $d=2$ is simpler). We do not have any space-time compactness of $\left(u_{n}\right)_{n \in \mathbb{N}}$ since we have no condition on $\partial_{t} u_{n}$. But, using the fact that $\left(\rho_{n}\right)_{n \in \mathbb{N}}$ is bounded in $L^{2}(] 0, T\left[, L^{\gamma}(\Omega)\right)$ and assuming that $\left(\boldsymbol{u}_{n}\right)_{n \in \mathbb{N}}$ is bounded in $L^{2}(] 0, T\left[, L^{6}(\Omega)\right)^{d}$ we deduce, using $\gamma>6 / 5$, that $\left(\partial_{t} \rho_{n}\right)_{n \in \mathbb{N}}$ is bounded in $L^{1}(] 0, T\left[, W^{-1,1}(\Omega)\right)$ and this gives a compactness result for the sequence $\left(\rho_{n}\right)_{n \in \mathbb{N}}$ in $L^{2}(] 0, T\left[, H^{-1}(\Omega)\right)$ by means of an adaptation of the well known compactness results for evolution equation due to J. L. Lions, J. P. Aubin and J. Simon (see, for instance, J.L. Lions [Lio69], J. P. Aubin [Aub63] and J. Simon [Sim87]). Indeed, one uses Theorem 1 with $B=H^{-1}(\Omega), X=L^{\gamma}(\Omega)$ and $Y=W^{-1,1}(\Omega)$ (note that $X$ is compactly embedded in $B$ since $\gamma>6 / 5$, this is due, by duality, to the fact that $H_{0}^{1}(\Omega)$ is compactly embedded in $L^{r}(\Omega)$ for $r<6)$. Then, one has $\rho_{n} \rightarrow \rho$ in $L^{2}(] 0, T\left[, H^{-1}(\Omega)\right)$. Since $\boldsymbol{u}_{n} \rightarrow \boldsymbol{u}$ weakly converges in $L^{2}(] 0, T\left[, H_{0}^{1}(\Omega)^{3}\right)$ we then obtain (16) and, similarly, since $\nabla \varphi$ is a 
regular function, (17). More precisely, for all $\psi \in C_{c}^{\infty}\left(\mathbb{R}^{3} \times\right] 0, T[)^{3}$, one has

$$
\begin{aligned}
\int_{0}^{T} \int_{\Omega} \rho_{n} \boldsymbol{u}_{n} \cdot \psi d x d t=\left\langle\rho_{n}, \boldsymbol{u}_{n} \cdot \psi\right\rangle_{L^{2}\left(H^{-1}\right), L^{2}\left(H_{0}^{1}\right)} & \\
\rightarrow\langle\rho, \boldsymbol{u} \cdot \psi\rangle_{L^{2}\left(H^{-1}\right), L^{2}\left(H_{0}^{1}\right)} & =\int_{0}^{T} \int_{\Omega} \rho \boldsymbol{u} \cdot \psi d x d t,
\end{aligned}
$$

which gives $\partial_{t} \rho+\operatorname{div}(\rho \boldsymbol{u})=0$ (in the distributional sense).

The same difficulty appears in the momentum equation in order to pass to the limit on $\operatorname{div}\left(\rho_{n} \boldsymbol{u}_{n} \otimes \boldsymbol{u}_{n}\right)$. This will be possible if one proves that for all $\psi \in C_{c}^{\infty}\left(\mathbb{R}^{3} \times\right] 0, T[)$, one has

$$
\lim _{n \rightarrow+\infty} \int_{0}^{T} \int_{\Omega} \rho_{n} v_{n} u_{n} \psi d x d t=\int_{0}^{T} \int_{\Omega} \rho v u \psi d x d t,
$$

where $u_{n}$ and $v_{n}$ are two components of $\boldsymbol{u}_{n}$ (and $u, v$ the corresponding components of $\boldsymbol{u}$ ).

We consider here also the case $d=3$ and we use the fact that $\gamma>3 / 2$. It gives that $\rho_{n} u_{n}$ is bounded in $L^{2}(] 0, T\left[, L^{r}(\Omega)\right)$ with $r=6 \gamma /(6+\gamma)>6 / 5$. Since we already know that $\rho_{n} u_{n} \rightarrow \rho u$ in the distributional sense, this gives that $\rho_{n} u_{n} \rightarrow$ $\rho u$ weakly in $L^{2}(] 0, T\left[, L^{r}(\Omega)\right)$. Using now the momentum equation, we prove that $\left(\partial_{t}\left(\rho_{n} u_{n}\right)\right)_{n \in \mathbb{N}}$ is bounded in $L^{1}(] 0, T\left[, W^{-1,1}(\Omega)\right)$ and this gives a compactness result for the sequence $\left(\rho_{n} u_{n}\right)_{n \in \mathbb{N}}$ in $L^{2}(] 0, T\left[, H^{-1}(\Omega)\right)$ using Theorem 1 with $B=H^{-1}(\Omega), X=L^{r}(\Omega)$ and $Y=W^{-1,1}(\Omega)$ ( $X$ is compactly embedded in $B$ since $r>6 / 5)$. Since $v_{n} \rightarrow v$ weakly converges in $L^{2}(] 0, T\left[, H_{0}^{1}(\Omega)\right)$ we then obtain (18) and this allows us to pass to the limit in $\operatorname{div}\left(\rho_{n} \boldsymbol{u}_{n} \otimes \boldsymbol{u}_{n}\right)$.

\subsubsection{Stefan problem}

Let $T>0, \Omega$ be a bounded open set of $\mathbb{R}^{d}, d \geq 1$. For $n \in \mathbb{N}$, let $\left(\rho_{n}, u_{n}\right)$ be a (weak) solution of (4) with an homogeneous Dirichlet boundary condition on $u_{n}$ and an initial condition on $\rho_{n}$ (bounded in $L^{2}(\Omega)$ ). We recall that $\varphi$ is a Lipschitz continuous function from $\mathbb{R}$ to $\mathbb{R}$, nondecreasing, such that $\varphi^{\prime}=0$ on ]$a, b\left[\right.$, for some real numbers $a, b$ with $a<b$ and $\liminf _{|s| \rightarrow+\infty}|\varphi(s)| /|s|>0$.

The natural estimates for this problem are an $L^{2}(] 0, T\left[, L^{2}(\Omega)\right)$-estimate on $\rho_{n}$ and an $L^{2}(] 0, T\left[, H_{0}^{1}(\Omega)\right)$-estimate on $u_{n}$. Then we have, up to a subsequence, $\rho_{n} \rightarrow \rho$ weakly in $L^{2}(] 0, T\left[, L^{2}(\Omega)\right)$ and $u_{n} \rightarrow u$ weakly in $L^{2}(] 0, T\left[, H_{0}^{1}(\Omega)\right)$. 
It is quite easy to pass to the limit in the equation $\partial_{t} \rho_{n}-\Delta u_{n}=0$ and one obtains $\partial_{t} \rho-\Delta u=0$, in the distributional sense, but it is less easy to prove that $u=\varphi(\rho)$.

The way to prove that $u=\varphi(\rho)$ consists in proving (16) and then to use the Minty trick given (in this simple case) in Lemma 1. In order to prove (16), one can use, as in Section 2.1, compactness on $\left(u_{n}\right)_{n \in \mathbb{N}}$ or compactness on $\left(\rho_{n}\right)_{n \in \mathbb{N}}$ (this was not the case in the case of the compressible Navier-Stokes equations described above).

The proof of compactness of $\left(u_{n}\right)_{n \in \mathbb{N}}$ in $L^{2}(] 0, T\left[, L^{2}(\Omega)\right)$ (which leads to (16)) is not easy since there is no direct estimate on $\partial_{t} u_{n}$, but a trick due to Alt and Luckhaus [AL83] allows to obtain directly an estimate on the time-translates of $u_{n}$ (without estimate on $\partial_{t} u_{n}$ ) in $L^{2}(] 0, T\left[, L^{2}(\Omega)\right)$ and therefore gives the compactness of $\left(u_{n}\right)_{n \in \mathbb{N}}$ in $L^{2}(] 0, T\left[, L^{2}(\Omega)\right)$. Then, (16) holds and this (with the Minty trick) concludes the proof of $u=\varphi(\rho)$.

Instead of proving compactness of $\left(u_{n}\right)_{n \in \mathbb{N}}$ in $L^{2}(] 0, T\left[, L^{2}(\Omega)\right)$, it is perhaps simpler to prove compactness of the sequence $\left(\rho_{n}\right)_{n \in \mathbb{N}}$ in $L^{2}(] 0, T\left[, H^{-1}(\Omega)\right)$. Indeed, since $\partial_{t} \rho_{n}-\Delta u_{n}=0$ and since $\left(u_{n}\right)_{n \in \mathbb{N}}$ is bounded in $L^{2}(] 0, T\left[, H_{0}^{1}(\Omega)\right)$, the sequence $\left(\partial_{t} \rho_{n}\right)_{n \in \mathbb{N}}$ is bounded in $L^{2}(] 0, T\left[, H^{-1}(\Omega)\right)$. This gives compactness of $\left(\rho_{n}\right)_{n \in \mathbb{N}}$ in $L^{2}(] 0, T\left[, H^{-1}(\Omega)\right)$ using Theorem 1 with $B=Y=H^{-1}(\Omega)$ and $X=L^{2}(\Omega)$. Then one has

$$
\begin{array}{r}
u_{n} \rightarrow u \text { weakly in } L^{2}(] 0, T\left[, H_{0}^{1}(\Omega)\right), \\
\rho_{n} \rightarrow \rho \text { in } L^{2}(] 0, T\left[, H^{-1}(\Omega)\right),
\end{array}
$$

and this gives (16) since

$$
\begin{aligned}
\int_{0}^{T} \int_{\Omega} \rho_{n} u_{n} d x d t=\left\langle\rho_{n}, u_{n}\right\rangle_{L^{2}\left(H^{-1}\right), L^{2}\left(H_{0}^{1}\right)} & \\
& \rightarrow\langle\rho, u\rangle_{L^{2}\left(H^{-1}\right), L^{2}\left(H_{0}^{1}\right)}
\end{aligned}
$$

Here also, it remains to use the Minty trick in order to conclude that $u=\varphi(\rho)$.

Remark 3. As for the stationary case, for this Stefan problem, it is also possible to use the compactness of $\left(\rho_{n}\right)_{n \in \mathbb{N}}$ under the form $\nabla w_{n} \rightarrow \nabla w$ in $L^{2}(] 0, T\left[, L^{2}(\Omega)^{d}\right)$ (and $\nabla u_{n} \rightarrow \nabla u$ weakly in $L^{2}(] 0, T\left[, L^{2}(\Omega)^{d}\right)$ ) where $w_{n}$ is the solution of (9) and $w$ is the solution of (10) (see, for instance, $\left[\mathrm{DEG}^{+} 17\right]$ ).

Lemma 1 (Minty trick). Let $Q$ be a bounded open set of $\mathbb{R}^{N}, N \geq 1$, and $\varphi$ be a continuous nondecreasing function from $\mathbb{R}$ to $\mathbb{R}$. We assume that there exists 
$C \in \mathbb{R}_{+}$such that

$$
|\varphi(s)| \leq C|s|+C \text { for all } s \in \mathbb{R} .
$$

(Note that the existence of $C$ is true if $\varphi$ is Lipschitz continuous.) Let $\left(\rho_{n}\right)_{n \in \mathbb{N}}$ and $\left(u_{n}\right)_{n \in \mathbb{N}}$ be sequences such that $\rho_{n} \rightarrow \rho$ weakly in $L^{2}(Q), u_{n} \rightarrow u$ weakly in $L^{2}(Q)$. We assume that $u_{n}=\varphi\left(\rho_{n}\right)$ a.e., for all $n$, and that

$$
\lim _{n \rightarrow+\infty} \int_{Q} \rho_{n}(y) u_{n}(y) d y=\int_{Q} \rho(y) u(y) d y .
$$

Then, $u=\varphi(\rho)$ a.e..

Proof. Since $\varphi$ is nondecreasing, one has, for all $\bar{\rho} \in L^{2}(Q)$,

$$
0 \leq \int_{Q}\left(\rho_{n}-\bar{\rho}\right)\left(\varphi\left(\rho_{n}\right)-\varphi(\bar{\rho})\right) d y=\int_{Q}\left(\rho_{n}-\bar{\rho}\right)\left(u_{n}-\varphi(\bar{\rho})\right) d y .
$$

Inequality (19) gives that $\varphi(\bar{\rho}) \in L^{2}(Q)$. Then,

$$
0 \leq \int_{Q} \rho_{n} u_{n} d y-\int_{Q} \rho_{n} \varphi(\bar{\rho}) d y-\int_{Q} \bar{\rho} u_{n} d y+\int_{Q} \bar{\rho} \varphi(\bar{\rho}) d y .
$$

Passing to the limit as $n \rightarrow+\infty$ in this inequality yieds

$$
0 \leq \int_{Q}(\rho-\bar{\rho})(u-\varphi(\bar{\rho})) d y .
$$

Let $\psi \in C_{c}^{\infty}(Q)$ and $m>0$. Taking $\bar{\rho}=\rho-(1 / m) \psi$ in the previous inequality gives

$$
0 \leq \int_{Q}(u-\varphi(\rho-(1 / m) \psi)) \psi d y
$$

The function $(u-\varphi(\rho-(1 / m) \psi)) \psi$ converges a.e. to $(u-\varphi(\rho) \psi$, as $m \rightarrow+\infty$, and is dominated by the fonction $(|u|+C|\rho|+C|\psi|+C) \psi$ which belongs to $L^{1}(Q)$. Then, using the Dominated Convergence Theorem, we obtain, as $m \rightarrow+\infty$,

$$
0 \leq \int_{Q}(u-\varphi(\rho)) \psi d y
$$

This inequality with $\psi$ and $-\psi$ gives $0=\int_{Q}(u-\varphi(\rho)) \psi d y$ and then, since $\psi$ is arbitrary, $u=\varphi(\rho)$ a.e. in $Q$.

In the previous examples, we use a compactness result for evolution equations which is essentially due to [Lio69, Aub63, Sim87], a proof is given in [GH15]. We now give this theorem. 
Theorem 1. Let $X, B, Y$ be three Banach spaces, $X \subset B, X \subset Y$, such that

1. $X$ is compactly embedded in $B$,

2. for any bounded sequence $\left(w_{n}\right)_{n \in \mathbb{N}}$ of $X$, if $\left\|w_{n}-w\right\|_{B} \rightarrow 0$ and $\left\|w_{n}\right\|_{Y} \rightarrow 0$, then $w=0$.

Let $T>0,1 \leq p<+\infty$ and $\left(u_{n}\right)_{n \in \mathbb{N}}$ be a sequence such that

- $\left(u_{n}\right)_{n \in \mathbb{N}}$ is bounded in $L^{p}(] 0, T[, X)$,

- $\left(\partial_{t} u_{n}\right)_{n \in \mathbb{N}}$ is bounded in $L^{1}(] 0, T[, Y)$.

Then there exists $u \in L^{p}(] 0, T[, B)$ such that, up to a subsequence, $u_{n} \rightarrow u$ in $L^{p}(] 0, T[, B)$.

Hypothesis 2 of Theorem 1 on the spaces, $X, B, Y$ is perhaps a little bit curious. Indeed, we can distinguish two cases. A simple situation appears when $\|\cdot\|_{B} \leq C\|\cdot\|_{Y}$ for some $C>0$ (this is the case, in particular, when $Y=X$ and Hypothesis 1 holds). Another situation is discussed in [Sim87], when $B$ is continuously embedded in $Y$, that is $\|\cdot\|_{Y} \leq C\|\cdot\|_{B}$ (for some $C>0$ ). Hypothesis 2 in Theorem 1 covers a more general framework.

An example of Banach spaces $X, B, Y$ satisfying $1-2$ is $X=W_{0}^{1,1}(\Omega), B=$ $L^{1}(\Omega), Y=W_{\star}^{-1,1}(\Omega)=\left(W_{0}^{1, \infty}(\Omega)\right)^{\prime}$, where, as usual, we identify an element of $L^{1}(\Omega)$ with the corresponding linear form on $W_{0}^{1, \infty}(\Omega)$.

A main tool, crucial for the case where $\|\cdot\|_{B} \not \leq C\|\cdot\|_{Y}$ is the following lemma essentially due to Lions [Lio69], the proof of which is quite easy by contradiction.

Lemma 2. Let $X, B, Y$ are three Banach spaces, $X \subset B, X \subset Y$, satisfying Hypotheses 1-2 of Theorem 1. Then, for any $\varepsilon>0$, there exists $C_{\varepsilon}$ such that, for $w \in X$,

$$
\|w\|_{B} \leq \varepsilon\|w\|_{X}+C_{\varepsilon}\|w\|_{Y} .
$$

A particular case for which the conclusion of Lemma 2 is true, simpler than Lemma 2, is when $B$ is a Hilbert space, $X$ is a Banach space continuously embedded in $B$ and $Y=X$ but the norm in $Y$, denoted by $\|\cdot\|_{Y}$, is the dual norm of $\|\cdot\|_{X}$ with respect to the scalar product of $B$, namely

$$
\|u\|_{Y}=\sup \left\{(u \mid v)_{B}, v \in X,\|v\|_{X} \leq 1\right\} .
$$

Then, for any $\varepsilon>0$ and $w \in X$, one has

$$
\|w\|_{B} \leq \varepsilon\|w\|_{X}+\frac{1}{\varepsilon}\|w\|_{Y}
$$

The proof is simple since

$$
\|u\|_{B}=(u \mid u)_{B}^{\frac{1}{2}} \leq\left(\|u\|_{Y}\|u\|_{X}\right)^{\frac{1}{2}} \leq \varepsilon\|w\|_{X}+\frac{1}{\varepsilon}\|w\|_{Y} .
$$

In this simple case, the compactness of $X$ in $B$ is not needed for the conclusion of Lemma 2 but nevertheless this compactness is needed for Theorem 1 even in 
this case. We can also remark that in this simple case, the space $Y$ is the space $X$ with the norm $\|\cdot\|_{Y}$ and it is not necessarily a Banach space. Indeed, the fact that $Y$ is a Banach space is never needed in Theorem 1, we only need $Y$ to be a normed space since we can always complete this normed space in order to have a Banach space.

We recall the spaces $X, B, Y$ which were used in this section for the examples describes above (Navier-Stokes Equations and Stefan Problem).

For the compressible Navier-Stokes equations, in order to prove the compactness of $\left(\rho_{n}\right)_{n \in \mathbb{N}}$ in $L^{2}(] 0, T\left[, H^{-1}(\Omega)\right)$ we choose $B=H^{-1}(\Omega), X=$ $L^{\gamma}(\Omega)$ and $Y=W^{-1,1}(\Omega)$. In order to prove the compactness of $\left(\rho_{n} u_{n}\right)_{n \in \mathbb{N}}$ in $L^{2}(] 0, T\left[, H^{-1}(\Omega)\right)$ we choose $B=H^{-1}(\Omega), X=L^{r}(\Omega)$ (with $r=6 \gamma /(6+\gamma)$ ) and $Y=W^{-1,1}(\Omega)$.

For the Stefan problem, we choose $X=L^{2}(\Omega), B=Y=H^{-1}(\Omega)$.

Remark 4. Another interesting example is the case of the incompressible NavierStokes equations for $d=2$ or 3 (see [CEGH14]), where we have to pass to the limit on $\operatorname{div}\left(\boldsymbol{u}_{n} \otimes \boldsymbol{u}_{n}\right)$. We set $H=\left\{\boldsymbol{u} \in H_{0}^{1}(\Omega)^{d}\right.$, $\left.\operatorname{div} \boldsymbol{u}=0\right\}$ (which is the natural space for the velocity) and we can use, for this case, Theorem 1 with $p=2, B=L^{2}(\Omega), X=H, Y=H^{\prime}$ (with the identification of $L^{2}(\Omega)$ with its dual space). It gives the compactness of the sequence of approximate velocities $\left(\boldsymbol{u}_{n}\right)_{n \in \mathbb{N}}$ in $L^{2}(] 0, T\left[, L^{2}(\Omega)^{d}\right)$ which allows to pass to the limit in $\operatorname{div}\left(\boldsymbol{u}_{n} \otimes \boldsymbol{u}_{n}\right)$. In this example one has $X$ continuously embedded in $B$ (and, even, compactly embedded in $B$ ) but $X$ is not dense in $B$ so that $B^{\prime}$ (which is identified with $B$ ) is not included in $Y$. However one has $H \subset H^{\prime}$ (using the identification of $B^{\prime}$ with $B$ ), which is the hypothesis needed in Theorem 1 . This is a situation quite general. If $E$ is a Banach space, continuously embedded in the Hilbert $F$, and if $F^{\prime}$ is identified with $F$, then one has $E \subset E^{\prime}$ but one does not have $F \subset E^{\prime}$ except if $E$ is dense in $F$ (see, for instance, [GH15, Chapter 4]).

\subsection{Discrete setting}

The objective of this section is to adapt the methods of Section 3.1 (in particular Theorem 1) to a discrete setting, in order to prove the convergence of numerical schemes.

The set $\Omega$ is now a bounded open set of $\mathbb{R}^{d}$ adapted to a space discretization. The time interval is $[0, T], T>0$.

Let $n \in \mathbb{N}$, one has a time step $k_{n}$ such that $T=k_{n} N_{n}$ with some positive integer $N_{n}$ and one has a space discretization which gives two finite dimensional spaces $L_{n}$ and $H_{n}$. As in the stationary case, Section 2.2, we consider the case 
where $L_{n}$ and $H_{n}$ are spaces of functions constant on control volumes defined by some meshes (which can be different for $L_{n}$ and $H_{n}$ as in interesting case of the MAC-scheme)

We assume that $\rho_{n}$ and $u_{n}$ are functions constant in time on each interval ]$(l-1) k_{n}, l k_{n}\left[\right.$, for $l=1, \ldots, N_{n}$ (but we could also assume that these functions are continuous in time and affine on each interval $](l-1) k_{n}, l k_{n}[$, this will not change the results given hereafter). For all $t \in](l-1) k_{n}, l k_{n}\left[, l=1, \ldots, N_{n}\right.$, one has

$$
\rho_{n}(\cdot, t)=\rho_{n}^{(l)} \in L_{n} \quad \text { and } \quad u_{n}(\cdot, t)=u_{n}^{(l)} \in H_{n} .
$$

Since we consider functions which are constant in time on each interval $](l-$ 1) $k_{n}, l k_{n}\left[\right.$, we have also to define discrete derivatives, namely for $l \in\left\{2, \ldots, N_{n}\right\}$,

$$
\begin{gathered}
\left.\partial_{t, n} u_{n}(\cdot, t)=\partial_{t, k_{n}}^{(l)} u=\frac{1}{k_{n}}\left(u_{n}^{(l)}-u_{n}^{(l-1)}\right) \text { for } t \in\right](l-1) k_{n}, l k_{n}[, \\
\left.\partial_{t, n} \rho_{n}(\cdot, t)=\partial_{t, k_{n}}^{(l)} \rho_{n}=\frac{1}{k_{n}}\left(\rho_{n}^{(l)}-\rho_{n}^{(l-1)}\right) \text { for } t \in\right](l-1) k_{n}, l k_{n}[, \\
\text { and, for } \left.l=1, \quad \partial_{t, n} u_{n}(\cdot, t)=\partial_{t, n} \rho_{n}(\cdot, t)=0, \text { for } t \in\right] 0, k_{n}[.
\end{gathered}
$$

We assume that $\lim _{n \rightarrow+\infty} k_{n}=0$ and $\lim _{n \rightarrow+\infty} h_{n}=0$, where $h_{n}$ is the maximum of the diameter of the control volumes of the meshes defining $L_{n}$ and $H_{n}$. The sequences $\left(\rho_{n}\right)_{n \in \mathbb{N}}$ and $\left(u_{n}\right)_{n \in \mathbb{N}}$ weakly converge to $\rho$ and $u$ in $L^{2}(] 0, T\left[, L^{2}(\Omega)\right)$ (we do not consider in this section the more general case of weak convergence in $L^{2}(] 0, T\left[, L^{q}(\Omega)\right)$ and $L^{2}(] 0, T\left[, L^{p}(\Omega)\right)$, with $\left.(1 / p)+(1 / q)=1\right)$. We want, as in Section 3.1, a convenient additional condition giving (16). We will mimic the method of Section 3.1.

We begin with a discrete version of Lemma 2 .

Lemma 3. Let $B$ be a Banach space and $\left(B_{n}\right)_{n \in \mathbb{N}}$ be a sequence of finite dimensional subspaces of $B$. Let $\|\cdot\|_{X_{n}}$ and $\|\cdot\|_{Y_{n}}$ be two norms on $B_{n}$ such that:

If $\left(\left\|w_{n}\right\|_{X_{n}}\right)_{n \in \mathbb{N}}$ is bounded, then,

1. up to a subsequence, there exists $w \in B$ such that $w_{n} \rightarrow w$ in $B$,

2. If $\left\|w_{n}-w\right\|_{B} \rightarrow 0$ and $\left\|w_{n}\right\|_{Y_{n}} \rightarrow 0$, then $w=0$.

Then, for any $\varepsilon>0$, there exists $\bar{C}_{\varepsilon}$ such that, for all $n \in \mathbb{N}$ and all $w \in B_{n}$,

$$
\|w\|_{B} \leq \varepsilon\|w\|_{X_{n}}+\bar{C}_{\varepsilon}\|w\|_{Y_{n}} .
$$

Proof. We first remark that $B_{n}$ is a finite dimensional subspace of $B$. Then, for all $n$ and for all $\varepsilon>0$, there exists $\bar{C}_{\varepsilon}$ satisfying (20) for all $u \in B_{n}$. The problem is to find $\bar{C}_{\varepsilon}$ independently of $n$.

We argue by contradiction. Assume that there exists $\varepsilon>0$ such that $\bar{C}_{\varepsilon}$ does not exists. Then, for a subsequence of $\left(B_{n}\right)_{n \in \mathbb{N}}$, still denoted $\left(B_{n}\right)_{n \in \mathbb{N}}$, there 
exists $\left(w_{n}\right)_{n \in \mathbb{N}}$ such that $w_{n} \in B_{n}$ and

$$
\left\|w_{n}\right\|_{B}>\varepsilon\left\|w_{n}\right\|_{X_{n}}+C_{n}\left\|w_{n}\right\|_{Y_{n}}
$$

with $\lim _{n \rightarrow+\infty} C_{n}=+\infty$.

By homogeneity, it is possible to assume that $\left\|w_{n}\right\|_{B}=1$. Then $\left(\left\|w_{n}\right\|_{X_{n}}\right)_{n \in \mathbb{N}}$ is bounded and, by Hypothesis 1, up to a subsequence, $w_{n} \rightarrow w$ in $B$ (so that $\|w\|_{B}=1$ ). But $\left\|w_{n}\right\|_{Y_{n}} \rightarrow 0$, so that $w=0$ (by Hypothesis 2), in contradiction with $\|w\|_{B}=1$.

We now give a discrete version of Theorem 1, the proof of which uses Lemma 3.

Theorem 2. Let $B$ be a Banach space, $1 \leq q<+\infty$ and $\left(B_{n}\right)_{n \in \mathbb{N}}$ a family of finite dimensional subspaces of $B$. Let $\|\cdot\|_{X_{n}}$ and $\|\cdot\|_{Y_{n}}$ be two norms on $B_{n}$ such that:

If $\left(\left\|w_{n}\right\|_{X_{n}}\right)_{n \in \mathbb{N}}$ is bounded, then,

1. up to a subsequence, there exists $w \in B$ such that $w_{n} \rightarrow w$ in $B$,

2. if $\left\|w_{n}-w\right\|_{B} \rightarrow 0$ and $\left\|w_{n}\right\|_{Y_{n}} \rightarrow 0$, then $w=0$.

The space $X_{n}$ is the space $B_{n}$ with norm $\|\cdot\|_{X_{n}}$, the space $Y_{n}$ is $B_{n}$ with norm $\|\cdot\|_{Y_{n}}$.

Let $T>0, k_{n}>0, T=k_{n} N_{n}$, and $\left(u_{n}\right)_{n \in \mathbb{N}}$ be a sequence such that

- for all $n, u_{n}(\cdot, t)=u_{n}^{(l)} \in B_{n}$ for $t \in\left((l-1) k_{n}, l k_{n}\right), l=1, \ldots, N_{n}$,

- the sequence $\left(u_{n}\right)_{n \in \mathbb{N}}$ is bounded in $L^{q}(] 0, T\left[, X_{n}\right)$, that is to say that there exists $C_{1}>0$ such that $\sum_{l=1}^{N_{n}} k_{n}\left\|u_{n}^{(l)}\right\|_{X_{n}}^{q} \leq C_{1}$ for all $n$,

- the sequence $\left(\partial_{t, k_{n}} u_{n}\right)_{n \in \mathbb{N}}$ is bounded in $L^{1}(] 0, T\left[, Y_{n}\right)$, that is to say that there exists $C_{2}>0$ such that $\sum_{l=2}^{N_{n}} k_{n}\left\|\partial_{t, k_{n}} u_{n}^{(l)}\right\|_{Y_{n}} \leq C_{2}$ for all $n$.

Then, there exists $u \in L^{q}(] 0, T[, B)$ such that, up to a subsequence, $u_{n} \rightarrow u$ in $L^{q}(] 0, T[, B)$.

See for instance [GH15, Theorem 4.51] for a proof of Theorem 2. Similar theorems are also in [CEGH14, GL12].

Of course, the main example for the present paper is $B_{n}=L_{n}$ or $B_{n}=H_{n}$. But it remains to choose $B,\|\cdot\|_{X_{n}}$ and $\|\cdot\|_{Y_{n}}$. We present these choices for the discretization of the two examples of Section 3.1, namely the compressible Navier-Sokes equations and the Stefan problem.

\subsubsection{Navier-Stokes Equations}

We begin with the case of the compressible Navier-Stokes equations as in Section 3.1.1 with a discretization using the MAC scheme, see [HW65, CEGH14]. In this example, $u_{n}$ is one component of the vector valued function $\boldsymbol{u}_{n}$, which is the 
discrete velocity field, and each component of $\boldsymbol{u}_{n}$ is a constant function on each control volume of its own mesh and for all time interval $](l-1) k_{n}, l k_{n}$ [ (it is the so-called staggered discretization). The discrete density $\rho_{n}$ is a constant function on the control volumes of another grid, generally called the primal grid, denoted $L_{n}$, and for all time interval $](l-1) k_{n}, l k_{n}[$.

We denote by $\bar{H}_{n}$ the spatial space for the discrete velocity field. This space is equipped with a norm, denoted $\|\cdot\|_{1,2, n}$, which mimics the $\left(H_{0}^{1}\right)^{d}$-norm. Indeed, this norm contains, for each component of $\boldsymbol{u}_{n}$ the norm $\|\cdot\|_{1,2, n}$ defined in Section 2.2 in the case of admissible meshes.

The estimates that can be obtained on the approximate solutions mimics the ones of the continuous setting. One obtain an estimate in $L^{\infty}(] 0, T\left[, L^{\gamma}(\Omega)\right)$ for $\rho_{n}$ and an estimate on $L^{2}(] 0, T\left[, \bar{H}_{n}\right)$ for $\boldsymbol{u}_{n}$ where $\bar{H}_{n}$ is equipped with the norm $\|\cdot\|_{1,2, n}$ (the way to obtain this estimate on $\boldsymbol{u}_{n}$ is roughly explained below). In particular this gives that the sequence $\left(\boldsymbol{u}_{n}\right)_{n \in \mathbb{N}}$ is bounded in $L^{2}(] 0, T\left[, L^{2}(\Omega)^{d}\right)$. We also assume that $\gamma \geq 2$ so that the sequence $\left(\rho_{n}\right)_{n \in \mathbb{N}}$ is bounded in $L^{2}(] 0, T\left[, L^{2}(\Omega)\right)$. Then, we can assume, up to a subsequence, that $\rho_{n} \rightarrow \rho$ weakly in $L^{2}(] 0, T\left[, L^{2}(\Omega)\right)$ and $\boldsymbol{u}_{n} \rightarrow \boldsymbol{u}$ weakly in $L^{2}(] 0, T\left[, L^{2}(\Omega)^{d}\right)$. We recall that we want to obtain (16) or, more generally, to prove the convergence of $\rho_{n} u_{n}$ to $\rho u$ in a convenient Lebesgue space. In order to obtain (16), we will use, as in Section 3.1.1, a compactness result on $\rho_{n}$.

Taking for instance, an implicit discretization of the mass balance (namely $\partial_{t} \rho+\operatorname{div}(\rho \boldsymbol{u})=0$ ), we have with some convenient upwind discretization of $\operatorname{div}(\rho \boldsymbol{u})$, for all $l \in\left\{2, \ldots, N_{n}\right\}$,

$$
\partial_{t, k_{n}}^{(l)} \rho_{n}+\operatorname{div}_{n}\left(\rho_{n}^{(l)} \boldsymbol{u}_{n}^{(l)}\right)=0 .
$$

A crucial idea in the discretization of Navier-Stokes Equations with staggered grids is to deduce from (21) a discrete mass balance on the mesh, or on the meshes (in the case of the MAC scheme), associated to the velocity field. In the case of the MAC scheme, this discrete mass balance reads for each $i=1, \ldots, d$ and for all $l \in\left\{2, \ldots, N_{n}\right\}$,

$$
\partial_{t, k_{n}}^{(l)} \rho_{n, i}+\operatorname{div}_{n, i}\left(\rho_{n}^{(l)} \boldsymbol{u}_{n}^{(l)}\right)=0,
$$

where the function $\rho_{n, i}$ is a reconstruction of $\rho_{n}$ on the mesh associated to the corresponding component of $\boldsymbol{u}_{\boldsymbol{n}}$ and $\operatorname{div}_{n, i}$ a discretization of div deduced from $\operatorname{div}_{n}$. We refer to [GGHL08, Section 3.3] for the first application of this idea, but not with the MAC scheme, and, for instance, [HL10] for the application of this idea with the MAC scheme. A main interest of this idea is that it gives, together with the discretization of the momentum equation, a kinetic energy balance and therefore an estimate on $\boldsymbol{u}_{n}$ in $L^{2}(] 0, T\left[, \bar{H}_{n}\right)$ where $\bar{H}_{n}$ is equipped with the norm $\|\cdot\|_{1,2, n}$. 
In order to apply Theorem 2 , one takes $B_{n}=L_{n}$ and $\|\cdot\|_{X_{n}}=\|\cdot\|_{L^{2}(\Omega)}$ so that $\left(\rho_{n}\right)_{n \in \mathbb{N}}$ is bounded in $L^{2}(] 0, T\left[, X_{n}\right)$. For the choice of $\|\cdot\|_{Y_{n}}$, we remark that a discrete integration by part leads to, for all $l \in\left\{2, \ldots, N_{n}\right\}$,

$$
\int_{\Omega} v \partial_{t, k_{n}}^{(l)} \rho_{n} d x=-\int_{\Omega} v \operatorname{div}_{n}\left(\rho_{n}^{(l)} \boldsymbol{u}_{n}^{(l)}\right) d x=\int_{\Omega}(\rho \boldsymbol{u})_{n}^{(l)} \cdot \nabla_{n} v d x, \text { for all } v \in L_{n},
$$

where $\nabla_{n}$ is a convenient discretization of $\nabla$. Then, a natural choice of $\|\cdot\|_{Y_{n}}$ is, for all $w \in L_{n}$,

$$
\|w\|_{Y_{n}}=\max \left\{\int_{\Omega} w \varphi ; \varphi \in L_{n} ;\left\|\nabla_{n} \varphi\right\|_{L^{\infty}(\Omega)^{d}}+\|\varphi\|_{L^{\infty}(\Omega)}=1\right\} .
$$

With this norm, one has $\left\|\partial_{t, k_{n}}^{(l)} \rho_{n}\right\|_{Y_{n}} \leq\left\|(\rho \boldsymbol{u})_{n}^{(l)}\right\|_{L^{1}(\Omega)^{d}}$. Since $\left(\rho_{n}\right)_{n}$ is bounded in $L^{2}(] 0, T\left[, L^{2}(\Omega)\right)$ and $\left(\boldsymbol{u}_{n}\right)_{n}$ is bounded in $L^{2}(] 0, T\left[, L^{2}(\Omega)^{d}\right)$, one has a bound for $(\rho \boldsymbol{u})_{n}$ in $L^{1}(] 0, T\left[, L^{1}(\Omega)^{d}\right)$ which gives a bound for $\partial_{t, k_{n}} \rho_{n}$ in $L^{1}(] 0, T\left[, Y_{n}\right)$.

We choose $B=\left(H^{s}\right)^{\prime}$ for $s$ such that $0<s<1 / 2$ (see Section 2.2 for the definition of $H^{s}$ ) and we now prove that the hypotheses 1-2 of Theorem 2 are satisfied. We already know that $L^{2}(\Omega)$ is compactly embedded in $\left(H^{s}\right)^{\prime}$ (see Section 2.2 and Lemma 5 in Section 4). This gives Hypothesis 1 . In order to prove Hypothesis 2 , let $w_{n} \in L_{n}$ such that $\left(w_{n}\right)_{n \in \mathbb{N}}$ is bounded in $L^{2}(\Omega), w_{n} \rightarrow w$ in $\left(H^{s}\right)^{\prime}$ and $\left\|w_{n}\right\|_{Y_{n}} \rightarrow 0$. We want to prove that $w=0$. Let $\varphi \in C_{c}^{\infty}(\Omega)$. We define $\varphi_{n}$ in $L_{n}$ taking, for instance, the values of $\varphi$ at the centers of the control volumes defining the space $L_{n}$. It is quite easy to prove that the $L^{\infty}$-norm of $\nabla_{n} \varphi_{n}$ is bounded by the $L^{\infty}$-norm of $\nabla \varphi$. Then, one has

$$
\begin{array}{r}
\left|\int_{\Omega} w_{n} \varphi_{n} d x\right| \leq\left\|w_{n}\right\|_{Y_{n}}\left(\left\|\nabla_{n} \varphi_{n}\right\|_{L^{\infty}(\Omega)^{d}}+\left\|\varphi_{n}\right\|_{L^{\infty}(\Omega)}\right) \\
\leq\left\|w_{n}\right\|_{Y_{n}}\left(\|\nabla \varphi\|_{L^{\infty}(\Omega)^{d}}+\|\varphi\|_{L^{\infty}(\Omega)}\right) .
\end{array}
$$

This gives that $\lim _{n \rightarrow+\infty} \int_{\Omega} w_{n} \varphi_{n} d x=0$. But, since $\left(w_{n}\right)_{n \in \mathbb{N}}$ is bounded in $L^{2}(\Omega)$ and $w_{n} \rightarrow w$ in $\left(H^{s}\right)^{\prime}$, one has also $w_{n} \rightarrow w$ weakly in $L^{2}(\Omega)$ (we use here the uniqueness of the limit in $\left.\left(H^{s}\right)^{\prime}\right)$. Then, since $\varphi_{n} \rightarrow \varphi$ uniformly in $\Omega$, one has

$$
\lim _{n \rightarrow+\infty} \int_{\Omega} w_{n} \varphi_{n} d x=\int_{\Omega} w \varphi d x .
$$

We then conclude that $\int_{\Omega} w \varphi d x=0$ for all $\varphi \in C_{c}^{\infty}(\Omega)$ and therefore that $w=0$ a.e. on $\Omega$.

We can now apply Theorem 2 , it gives $\rho_{n} \rightarrow \rho$ in $L^{2}(] 0, T\left[,\left(H^{s}\right)^{\prime}\right)$. 
Indeed, the previous proof shows that it is also possible to prove that the sequence $\left(\partial_{t, k_{n}} \rho_{n}\right)_{n \in \mathbb{N}}$ is bounded in $L^{1}(] 0, T\left[, W_{\star}^{-1,1}(\Omega)\right)$ and then to apply Theorem 2 with $\|\cdot\|_{Y_{n}}=\|\cdot\|_{W_{\star}^{-1,1}(\Omega)}$, where $W_{\star}^{-1,1}(\Omega)=W_{0}^{1, \infty}(\Omega)^{\prime}$.

We now conclude. Taking $0<s<1 / 2$, we recall, see Section 2.2 and Lemma 4 , that an estimate on $\|v\|_{1,2, n}$ gives an estimate on $v$ in $H^{s}$. Then, the sequence $\left(\boldsymbol{u}_{\boldsymbol{n}}\right)_{n \in \mathbb{N}}$ is bounded in $L^{2}(] 0, T\left[,\left(H^{s}\right)^{d}\right)$ and therefore weakly convergent in this space (up to a subsequence). By uniqueness of the weak limit in $L^{2}(] 0, T\left[, L^{2}(\Omega)^{d}\right)$, its limit is necessarily $\boldsymbol{u}$ (and the convergence holds without extracting a subsequence) so that we finally obtain (16) for any component $u_{n}$ of $\boldsymbol{u}_{n}$. Furthermore it is possible to prove that for all $\varphi \in C^{\infty}\left(\mathbb{R}^{d} \times\right] 0, T[, \mathbb{R})$ one has

$$
\begin{aligned}
\int_{0}^{T} \int_{\Omega} \rho_{n} \boldsymbol{u}_{n} \cdot \nabla_{n} \varphi d x d t & =\left\langle\rho_{n}, \boldsymbol{u}_{n} \cdot \nabla \varphi\right\rangle_{L^{2}\left(\left(H^{s}\right)^{\prime}\right), L^{2}\left(H^{s}\right)}+R_{n} \\
& \rightarrow\langle\rho, \boldsymbol{u} \cdot \nabla \varphi\rangle_{L^{2}\left(\left(H^{s}\right)^{\prime}\right), L^{2}\left(H^{s}\right)}=\int_{0}^{T} \int_{\Omega} \rho \boldsymbol{u} \cdot \nabla \varphi d x d t .
\end{aligned}
$$

This gives, in particular, $\partial_{t} \rho+\operatorname{div}(\rho \boldsymbol{u})=0$ in the distributional sense.

The previous proof gives also, for all $\psi \in C^{\infty}\left(\mathbb{R}^{d} \times\right] 0, T\left[, \mathbb{R}^{d}\right)$,

$$
\int_{0}^{T} \rho_{n} \boldsymbol{u}_{n} \psi d x d t \rightarrow \int_{0}^{T} \rho \boldsymbol{u} \psi d x d t .
$$

In the case $d=3$ (the most interesting case), one has an estimate on $\boldsymbol{u}_{n}$ in $L^{2}(] 0, T\left[, L^{6}(\Omega)^{d}\right)$ (this is due to the discrete Sobolev inequality, see Section 2.1, proven in [EGH00, Lemma 3.5]) and then an estimate on $\rho_{n} \boldsymbol{u}_{n}$ in $L^{2}(] 0, T\left[, L^{r}(\Omega)^{d}\right)$. with $r=6 \gamma /(6+\gamma)$. This allows us to concude that $\rho_{n} \boldsymbol{u}_{n} \rightarrow \rho \boldsymbol{u}$ weakly in $L^{2}(] 0, T\left[, L^{r}(\Omega)\right)$. If $\gamma \geq 3$, one has $r \geq 2$. Then it is also possible, if $\gamma \geq 3$, to pass to the limit (in the momentum equation) in the term $\operatorname{div}\left(\rho_{n} \boldsymbol{u}_{n} \otimes \boldsymbol{u}_{n}\right)$ as we did in Section 3.1.1. We use Theorem 2 with each component of $\boldsymbol{u}_{n}$ and the corresponding reconstruction of $\rho_{n}$ on the associated mesh (and this gives the space $B_{n}$ ). We choose $B=\left(H^{s}\right)^{\prime}($ with $0<s<1 / 2$ ), the $L^{2}$-norm for $X_{n}$ and, for instance, the $W_{\star}^{-1,1}(\Omega)$-norm for $Y_{n}$. This gives the compactness in $L^{2}(] 0, T\left[,\left(H^{s}\right)^{\prime}\right)$ for each component of the sequence $\left(\rho_{n} \boldsymbol{u}_{n}\right)_{n \in \mathbb{N}}$ and we conclude using the boundedness in $L^{2}(] 0, T\left[, H^{s}\right)$ of each component of the sequence $\left(\boldsymbol{u}_{n}\right)_{n \in \mathbb{N}}$. 


\subsubsection{Stefan Problem}

For the case of the Stefan problem, the spatial discretization is the same for $\rho_{n}$ and for $u_{n}$ (with the notation of the beginning of Section 3.2, one has $L_{n}=H_{n}$ ). We recall that we are interested by the case where the space $H_{n}$ is not included in $H_{0}^{1}(\Omega)$ but $H_{n}$ is equipped with a norm, depending on $n$, "close" to the $H_{0}^{1}$-norm and denoted $\|\cdot\|_{1,2, n}$. We refer, for instance, to [EGH00, EGH10, DEG $\left.{ }^{+} 17\right]$.

The discretization of the Stefan problem described in Section 1, gives that the couple $\left(\rho_{n}, u_{n}\right)$ satisfy

$$
\partial_{t, k_{n}} \rho_{n}-\Delta_{n} u_{n}=0, u_{n}=\varphi\left(\rho_{n}\right)
$$

The discrete operator $\Delta_{n}$ from $H_{n}$ to $H_{n}$ is a convenient discretization of $\Delta$ as it is done, for instance, in [EGH00, EGH10, DEG $\left.{ }^{+} 17\right]$. We recall that $\varphi$ is a Lipschitz continuous function from $\mathbb{R}$ to $\mathbb{R}$, nondecreasing such that $\varphi^{\prime}=0$ on ]$a, b\left[\right.$, for some real numbers $a, b$ with $a<b$ and $\liminf _{|s| \rightarrow+\infty}|\varphi(s)| /|s|>0$.

A natural estimate for this problem gives that the sequence $\left(u_{n}\right)_{n \in \mathbb{N}}$ is bounded in $L^{2}(] 0, T\left[, Z_{n}\right)$, where $Z_{n}$ is the space $H_{n}$ with the norm $\|\cdot\|_{1,2, n}$. Then, using a discrete Poincaré estimate (see [EGH00] for the case of admissible meshes or [EGH10] for a more general case of meshes), one obtains that the sequence $\left(u_{n}\right)_{n \in \mathbb{N}}$ is bounded in $L^{2}(] 0, T\left[, L^{2}(\Omega)\right)$. From this estimate on $u_{n}$, one deduces, with the hypothesis $\lim \inf _{|s| \rightarrow+\infty}|\varphi(s)| /|s|>0$, that the sequence $\left(\rho_{n}\right)_{n \in \mathbb{N}}$ is also bounded in $L^{2}(] 0, T\left[, L^{2}(\Omega)\right)$. A consequence of these estimates is that we can assume, up to a subsequence, that $\rho_{n} \rightarrow \rho$ weakly in $L^{2}(] 0, T\left[, L^{2}(\Omega)\right)$ and $u_{n} \rightarrow u$ weakly in $L^{2}(] 0, T\left[, L^{2}(\Omega)\right)$.

The weak convergence of $\rho_{n}$ and $u_{n}$ and some consistency property of the discretization of $\Delta$ lead to

$$
\partial_{t} \rho-\Delta u=0
$$

Using the estimate of $u_{n}$ is $L^{2}(] 0, T\left[, Z_{n}\right)$, it is also classical to prove that $u \in L^{2}(] 0, T\left[, H_{0}^{1}(\Omega)\right)$ (see [EGH00] for the case of admissible meshes or [EGH10] for a more general case of meshes).

Our purpose here is to give two ways to prove that $u=\varphi(\rho)$. As in the continuous case, the first step is to prove (16) and then to conclude with the Minty trick (Lemma 1).

For proving (16), as in the continuous case (Section 3.1.2), we can use compactness of $\left(u_{n}\right)_{n \in \mathbb{N}}$ or compactness of $\left(\rho_{n}\right)_{n \in \mathbb{N}}$.

The compactness of $\left(u_{n}\right)_{n \in \mathbb{N}}$ in not given by an application of Theorem 2 because we do not have any estimate on $\partial_{t, k_{n}} u_{n}$. However, it is sometimes possible to adapt the method of Alt-Luckhaus [AL83] to this discrete setting in order to obtain some estimates on the time-translates of $u_{n}$ and then compactness 
of the sequence $\left(u_{n}\right)_{n \in \mathbb{N}}$ in $L^{2}(] 0, T\left[, L^{2}(\Omega)\right)$. This gives (16) and then, using the Minty trick, $u=\varphi(\rho)$. This way is, for instance, used in [EGH00].

The second way is to prove some compactness on the sequence $\left(\rho_{n}\right)_{n \in \mathbb{N}}$. We recall that the norm $\|\cdot\|_{1,2, n}$ control the $H^{s}$-norm for some convenient $s>0$ (see Section 2.2 and Lemma 4 in Section 4). In the case of admissible meshes as in [EGH00], we can take any $s<1 / 2$. in the case of more general meshes as in [EGH10], we can take, for $d=3$, any $s<2 / 5$. Then, one has $u_{n} \rightarrow u$ weakly in $L^{2}(] 0, T\left[, H^{s}\right)$ (since $L^{2}(] 0, T\left[, H^{s}\right)$ is a Hilbert space). In order to prove (16), it suffices to prove that $\left(\rho_{n}\right)_{n \in \mathbb{N}}$ converges in $L^{2}(] 0, T\left[,\left(H^{s}\right)^{\prime}\right)$ (as usual, $L^{2}(\Omega)$ is identified with its dual space). We will prove this compactness on $\left(\rho_{n}\right)_{n \in \mathbb{N}}$ with Theorem 2 applied with

$$
B=\left(H^{s}\right)^{\prime}, B_{n}=H_{n},\|\cdot\|_{X_{n}}=\|\cdot\|_{L^{2}(\Omega)},\|\cdot\|_{Y_{n}}=\|\cdot\|_{-1,2, n},
$$

where $\|\cdot\|_{-1,2, n}$ is the dual norm of the norm $\|\cdot\|_{1,2, n}$, that is, for $v \in H_{n}$,

$$
\|v\|_{-1,2, n}=\max \left\{\int_{\Omega} v w d x ; w \in H_{n},\|w\|_{1,2, n}=1\right\} .
$$

Multiplying, for $w \in H_{n}$, the equation $\partial_{t, k_{n}} \rho_{n}-\Delta_{n} u_{n}=0$ by $w$ and using a discrete integration by part, the estimate on $u_{n}$ in $L^{2}(] 0, T\left[, Z_{n}\right)$ gives an estimate on $\partial_{t, k_{n}} \rho_{n}$ in $L^{2}(] 0, T\left[, Y_{n}\right)$. In order to apply Theorem 2 , it remains to verify Hypotheses 1-2 of Theorem 2. Hypothesis 1 is due to compactness of $L^{2}(\Omega)$ in $\left(H^{s}\right)^{\prime}$ (which is a consequence, by duality, of the compact embedding of $H^{s}$ in $L^{2}(\Omega)$, Lemma 5 in Section 4$)$. For proving Hypothesis 2 , let $\left(w_{n}\right)_{n \in \mathbb{N}}$ be a bounded sequence of $L^{2}(\Omega)$ converging to $w$ in $\left(H^{s}\right)^{\prime}$ and to 0 for the $Y_{n^{-}}$ norm. We first remark that $w_{n} \rightarrow w$ weakly in $L^{2}(\Omega)$. Let $\psi \in C_{c}^{\infty}(\Omega)$. We can define $\psi_{n}$ in $L_{n}$ taking for instance the mean values of $\psi$ on the control volumes defining the space $L_{n}$. It is possible to prove that $\left\|\psi_{n}\right\|_{1,2, n} \leq C\|\psi\|_{H^{1}(\Omega)}$ where $C$ depends only the regularity of the mesh (see, for instance, [EGH00, Lemma 9.4] for the case of admissible meshes). Then, under a regularity hypothesis on the sequence of meshes, one has

$$
\left|\int_{\Omega} w_{n} \psi_{n} d x\right| \leq\left\|w_{n}\right\|_{Y_{n}}\left\|\psi_{n}\right\|_{1,2, n} \leq C\left\|w_{n}\right\|_{Y_{n}}\|\psi\|_{H^{1}(\Omega)} .
$$

This gives that $\lim _{n \rightarrow+\infty} \int_{\Omega} w_{n} \psi_{n} d x=0$. But, since $w_{n} \rightarrow w$ weakly in $L^{2}(\Omega)$ and $\psi_{n} \rightarrow \psi$ uniformly in $\Omega$, one has

$$
\lim _{n \rightarrow+\infty} \int_{\Omega} w_{n} \psi_{n} d x=\int_{\Omega} w \psi d x
$$


We then conclude that $\int_{\Omega} w \psi d x=0$ for all $\psi \in C_{c}^{\infty}(\Omega)$ and therefore that $w=0$ a.e. on $\Omega$.

All the hypotheses of Theorem 2 are satisfied and we obtain the convergence of $\left(\rho_{n}\right)_{n \in \mathbb{N}}$ in $L^{2}(] 0, T\left[,\left(H^{s}\right)^{\prime}\right)$. It gives (16) and we conclude with the Minty trick (Lemma 1) that $u=\varphi(\rho)$.

Remark 5. Here also, as in Section 2.2, it is possible to use the compactness of $\left(\rho_{n}\right)_{n \in \mathbb{N}}$ under the form $\nabla_{n} w_{n} \rightarrow \nabla w$ in $L^{2}(] 0, T\left[, L^{2}(\Omega)^{d}\right)$ (and $\nabla_{n} u_{n} \rightarrow \nabla u$ weakly in $\left.L^{2}(] 0, T\left[, L^{2}(\Omega)^{d}\right)\right)$ where $\nabla_{n}$ is a discretization of $\nabla, w_{n}$ is the solution of a discrete equivalent of $(9)$ and $w$ is the solution of (10). This method is used, for instance, in $\left[\mathrm{DEG}^{+} 17\right]$.

\section{Appendix}

We prove here that the space $N_{\alpha, 2}\left(\mathbb{R}^{d}\right)$ (which is a Nikolsky-space) is continuously embedded in the space $H^{s}\left(\mathbb{R}^{d}\right)$ for $0 \leq s<\alpha \leq 1$ (Lemma 4) and that the space $H^{s}$ (with $s>0$ and $\Omega$ bounded) is compactly embedded in $L^{2}\left(\mathbb{R}^{d}\right)$ (Lemma 5).

Definition 2. Let $0<\alpha \leq 1$ and $d \geq 1$. The space $N_{\alpha, 2}\left(\mathbb{R}^{d}\right)$ is the set of elements $u$ in $L^{2}(\Omega)$ such that there exists $C$ satisfying

$$
\|u(\cdot+\eta)-u\|_{L^{2}\left(\mathbb{R}^{d}\right)} \leq C|\eta|^{\alpha}, \text { for all } \eta \in \mathbb{R}^{d} .
$$

The norm in the space $N_{\alpha, 2}\left(\mathbb{R}^{d}\right)$ is defined by

$$
\|u\|_{N_{\alpha, 2}}=\|u\|_{L^{2}\left(\mathbb{R}^{d}\right)}+\max _{\eta \in \mathbb{R}^{d}, \eta \neq 0} \frac{\|u(\cdot+\eta)-u\|_{L^{2}\left(\mathbb{R}^{d}\right)}}{|\eta|^{\alpha}} .
$$

With this norm, $N_{\alpha, 2}$ is a Banach space.

Lemma 4. Let $d \geq 1,0 \leq s<\alpha \leq 1$. Then the space $N_{\alpha, 2}\left(\mathbb{R}^{d}\right)$ is continuously embedded in the space $H^{s}\left(\mathbb{R}^{d}\right)$ (see Definition 1).

Proof. Let $u \in N_{\alpha, 2}\left(\mathbb{R}^{d}\right)$ and $C=\|u\|_{N_{\alpha, 2}}$. For all $\eta \in \mathbb{R}^{d}$, one has

$$
\|\widehat{u(\cdot+\eta)}-\widehat{u}\|_{L^{2}\left(\mathbb{R}^{d}\right)}=\|u(\cdot+\eta)-u\|_{L^{2}\left(\mathbb{R}^{d}\right)} \leq C|\eta|^{\alpha} .
$$

We recall that for all $\eta \in \mathbb{R}^{d}, \widehat{u(\cdot+\eta)}(\xi)=e^{i \eta \cdot \xi} \hat{u}(\xi)$ a.e.. Then

$$
\int_{\mathbb{R}^{d}}\left|e^{i \eta \cdot \xi}-1\right|^{2}|\widehat{u}(\xi)|^{2} d \xi \leq C^{2}|\eta|^{2 \alpha} .
$$


Let $e_{1}, \ldots, e_{d}$ be the canonical basis of $\mathbb{R}^{d}$. Let $j \in\{1, \ldots, d\}$ and $t>0$. With $\eta=t e_{j}$ in (25) one obtains

$$
\int_{\mathbb{R}^{d}} \frac{\left|e^{i t \xi_{j}}-1\right|^{2}}{t^{2 \alpha}}|\widehat{u}(\xi)|^{2} d \xi \leq C^{2},
$$

and then, for $\varepsilon>0$ and for all $t>0$,

$$
\int_{\mathbb{R}^{d}} \frac{\left|e^{i t \xi_{j}}-1\right|^{2}}{t^{2 \alpha}}|\widehat{u}(\xi)|^{2} d \xi \frac{1}{t^{1-\varepsilon}} \leq C^{2} \frac{1}{t^{1-\varepsilon}} .
$$

Integrating this inequality between 0 and 1 and using Fubini-Tonelli Theorem lead to

$$
\int_{\mathbb{R}^{d}}|\widehat{u}(\xi)|^{2}\left(\int_{0}^{1} \frac{\left|e^{i t \xi_{j}}-1\right|^{2}}{t^{2 \alpha+1-\varepsilon}} d t\right) d \xi \leq \frac{C^{2}}{\varepsilon} .
$$

We use now the change of variable $t\left|\xi_{j}\right|=\tau$ so that (since $\left|e^{i \tau}-1\right|=\left|e^{-i \tau}-1\right|$ )

$$
\int_{\mathbb{R}^{d}}|\widehat{u}(\xi)|^{2}\left|\xi_{j}\right|^{2 \alpha-\varepsilon}\left(\int_{0}^{\left|\xi_{j}\right|} \frac{\left|e^{i \tau}-1\right|^{2}}{\tau^{2 \alpha+1-\varepsilon}} d \tau\right) d \xi \leq \frac{C^{2}}{\varepsilon} .
$$

We set $a_{\varepsilon}=\int_{0}^{1} \frac{\left|e^{i \tau}-1\right|^{2}}{\tau^{2 \alpha+1-\varepsilon}} d \tau$.

Note that $0<a_{\varepsilon}<+\infty$ since $\left|e^{i \tau}-1\right|^{2}=(\cos (\tau)-1)^{2}+(\sin (\tau))^{2} \leq 2 \tau^{2}$ and $\alpha \leq 1$. With this definition of $a_{\varepsilon}$, one has

$$
\begin{aligned}
& \int_{\mathbb{R}^{d}}|\widehat{u}(\xi)|^{2}\left|\xi_{j}\right|^{2 \alpha-\varepsilon} d \xi \\
& \quad \leq \int_{\left|\xi_{j}\right|<1}|\widehat{u}(\xi)|^{2} d \xi+\frac{1}{a} \int_{\left|\xi_{j}\right| \geq 1}|\widehat{u}(\xi)|^{2}\left|\xi_{j}\right|^{2 \alpha-\varepsilon}\left(\int_{0}^{\left|\xi_{j}\right|} \frac{\left|e^{i \tau}-1\right|^{2}}{\tau^{2 \alpha+1-\varepsilon}} d \tau\right) d \xi,
\end{aligned}
$$

and then we deduce from (26)

$$
\int_{\mathbb{R}^{d}}|\widehat{u}(\xi)|^{2}\left|\xi_{j}\right|^{2 \alpha-\varepsilon} d \xi \leq\|u\|_{L^{2}\left(\mathbb{R}^{d}\right)}^{2}+\frac{C^{2}}{\varepsilon a_{\varepsilon}} \leq b_{\varepsilon}\|u\|_{N_{\alpha, 2}}^{2},
$$

with $b_{\varepsilon}=1+\frac{1}{\varepsilon a_{\varepsilon}}$. This gives

$$
\|u\|_{\alpha-\frac{\varepsilon}{2}}^{2}=\int_{\mathbb{R}^{d}}\left(1+|\xi|^{2}\right)^{\alpha-\frac{\varepsilon}{2}}|\widehat{u}(\xi)|^{2} d \xi \leq d b_{\varepsilon}\|u\|_{N_{\alpha, 2}}^{2}
$$

and concludes the proof. 
Lemma 5. Let $\Omega$ be a bounded open set of $\mathbb{R}^{d}(d \geq 1), s>0$ and

$$
H^{s}=\left\{u \in H^{s}\left(\mathbb{R}^{d}\right), u=0 \text { a.e. on } \mathbb{R}^{d} \backslash \Omega\right\},
$$

equipped with the norm $\|\cdot\|_{s}$ given in (13). Then, $H^{s}$ is compactly embedded in $L^{2}\left(\mathbb{R}^{d}\right)$.

Proof. Since $H^{\bar{s}}$ is continuously embedded in $H^{s}$ for $0 \leq s<\bar{s}$, we have only to prove the lemma for $0<s \leq 1$.

Let $0<s \leq 1$, we are going to prove that for all $u \in H^{s}$ and $\eta \in \mathbb{R}^{d}$,

$$
\|u(\cdot+\eta)-u\|_{L^{2}\left(\mathbb{R}^{d}\right)} \leq 2\|u\|_{s}|\eta|^{s}
$$

Inequality (27) gives that $H^{s}$ is compactly embedded in $L^{2}\left(\mathbb{R}^{d}\right)$. It is a consequence of the Kolmogorov theorem in $L^{p}$-spaces (see, for instance, [GH13, Theorem 8.16]).

Let $\eta \in \mathbb{R}^{d}, \eta \neq 0$. One has, as in Lemma 4 ,

$$
\|u(\cdot+\eta)-u\|_{L^{2}\left(\mathbb{R}^{d}\right)}^{2}=\|\widehat{u(\cdot+\eta)}-\widehat{u}\|_{L^{2}\left(\mathbb{R}^{d}\right)}^{2}=\int_{\mathbb{R}^{d}}\left|e^{i \eta \cdot \xi}-1\right|^{2}|\widehat{u}(\xi)|^{2} d \xi .
$$

We now remark that for all $\eta, \xi \in \mathbb{R}^{d}$, one has

$$
\left|e^{i \eta \cdot \xi}-1\right|^{2} \leq 4\left(1+|\xi|^{2}\right)^{s}|\eta|^{2 s} .
$$

Indeed, since (for all $x \in \mathbb{R}$ ) $\left|e^{i x}-1\right| \leq 2,(28)$ is true if $|\eta| \geq 1$ and if $|\eta|<1$ but $|\xi||\eta| \geq 1$. If $|\eta|<1$ and $|\xi||\eta|<1$, (28) is also true since $\left|e^{i x}-1\right|^{2} \leq 2 x^{2}$ and then $\left|e^{i \eta \cdot \xi}-1\right|^{2} \leq 2|\xi|^{2}|\eta|^{2} \leq 2|\xi|^{2 s}|\eta|^{2 s}$ (since $s \leq 1$ ), which gives (28). Using (28), we then obtain that

$$
\|u(\cdot+\eta)-u\|_{L^{2}\left(\mathbb{R}^{d}\right)}^{2} \leq \int_{\mathbb{R}^{d}} 4\left(1+|\xi|^{2}\right)^{s}|\eta|^{2 s}|\widehat{u}(\xi)|^{2} d \xi
$$

and, finally, $\|u(\cdot+\eta)-u\|_{L^{2}\left(\mathbb{R}^{d}\right)} \leq 2\|u\|_{s}|\eta|^{s}$. This concludes the proof.

\section{Conclusion}

This paper presents some tools useful for proving the convergence of numerical schemes. The main result is Theorem 2. Two applications are presented. The first one appears for the discretization of the Navier-Stokes equations and the second 
one for the Stefan problem. However, one may find other problems where the tools discussed here can be applied for proving the convergence of the numerical schemes. The ideas that are developed in this paper can probably also been extended to the analysis of high-order nonconforming methods (e.g., for the convergence analysis of discretization schemes for the Navier-Stokes equations, Leray-Lions problems, nonlinear elasticity models... ).

In the case of the discrete setting of the compressible Navier-Stokes equations when the equation of state does not allow an $L^{2}(] 0, T\left[, L^{2}(\Omega)\right)$-estimate on $\rho$, it will be interesting to work with a sequence $\left(\rho_{n}\right)_{n \in \mathbb{N}}$ weakly convergent in $L^{2}(] 0, T\left[, L^{q}(\Omega)\right)$ with some $1<q<+\infty$ and a sequence $\left(u_{n}\right)_{n \in \mathbb{N}}$ weakly convergent in $L^{2}(] 0, T\left[, L^{p}(\Omega)\right)$ with $(1 / p)+(1 / q)=1$ (we only consider in this paper the case $p=q=2)$.

\section{References}

[AL83] Hans Wilhelm Alt and Stephan Luckhaus. Quasilinear ellipticparabolic differential equations. Math. Z., 183(3):311-341, 1983.

[Aub63] Jean-Pierre Aubin. Un théorème de compacité. C. R. Acad. Sci. Paris, 256:5042-5044, 1963.

[CEGH14] Eric Chénier, Robert Eymard, Thierry Gallouët, and Raphaèle Herbin. An extension of the MAC scheme to locally refined meshes: convergence analysis for the full tensor time-dependent Navier-Stokes equations. Calcolo, pages 1-39, 2014.

[DEG $\left.{ }^{+} 17\right]$ Jérôme Droniou, Robert Eymard, Thierry Gallouët, Cindy Guichard, and Raphaele Herbin. The gradient discretisation method: A framework for the discretisation and numerical analysis of linear and nonlinear elliptic and parabolic problems. Version 4, November 2017. URL: https://hal.archives-ouvertes.fr/hal-01382358.

[EGH00] R. Eymard, T. Gallouët, and R. Herbin. Finite volume methods. Ciarlet, P. G. (ed.) et al., in Handbook of numerical analysis. NorthHolland, Amsterdam, pp. 713-1020, 2000.

[EGH10] R. Eymard, T. Gallouët, and R. Herbin. Discretization of heterogeneous and anisotropic diffusion problems on general nonconforming meshes SUSHI: a scheme using stabilization and hybrid interfaces. IMA J. Numer. Anal., 30(4):1009-1043, 2010.

[Gal17] Thierry Gallouët. Some discrete functional analysis tools. In Finite volumes for complex applications. VIII, Springer Proc. Math. Stat. Springer, 2017. 
[GGHL08] Thierry Gallouët, Laura Gastaldo, Raphaele Herbin, and Jean-Claude Latché. An unconditionally stable pressure correction scheme for the compressible barotropic Navier-Stokes equations. M2AN Math. Model. Numer. Anal., 42(2):303-331, 2008.

[GH13] Thierry Gallouët and Raphaele Herbin. Mesure, Intégration, Probabilités. Ellipse, Paris, 2013.

[GH15] Thierry Gallouët and Raphaele Herbin. Equations aux dérivées partielles. Lecture, September 2015. URL: https://hal.archivesouvertes.fr/cel-01196782.

[GHLM17] T. Gallouët, R. Herbin, J.-C. Latché, and D. Maltese. Convergence of the MAC scheme for the compressible stationary Navier-Stokes equations. Mathematics of Computation, published electronically: September 19, 2017.

[GL12] T. Gallouët and J.-C. Latché. Compactness of discrete approximate solutions to parabolic PDEs - application to a turbulence model. Commun. Pure Appl. Anal., 11(6):2371-2391, 2012.

[HL10] R. Herbin and J.-C. Latché. Kinetic energy control in the MAC discretization of the compressible Navier-Stokes equations. Int. J. Finite Vol., 7(2):6, 2010.

[HW65] F.H. Harlow and J.E. Welch. Numerical calculation of time dependent viscous incompressible flow of fluids with free surface. Phys. Fluids, 8:2182-2189, 1965.

[Lio69] J.-L. Lions. Quelques méthodes de résolution des problèmes aux limites non linéaires. Dunod, Paris, 1969.

[Sim87] J. Simon. Compact sets in the space Lp(0,T;B). Annali di Matematica Pura ed Applicata, 146:65-96, 1987. 